\title{
Rapid solution for analysis of nonlinear fluid film force and dynamic behavior of a tilting-pad journal bearing-rotor system with turbulent and thermal effects
}

\author{
Yingze JIN, Zhaoyang SHI, Xiaojing ZHANG, Xiaoyang YUAN* \\ Key Laboratory of the Education Ministry for Modern Design and Rotor-Bearing Systems, Xi'an Jiaotong University, Xi'an 710049, China \\ Received: 11 April 2018 / Revised: 03 June 2018 / Accepted: 07 December 2018 \\ (C) The author(s) 2019.
}

\begin{abstract}
To analyze the nonlinear dynamics of a tilting-pad journal bearing (TPJB)-rotor system with high accuracy and speed, the database method (DM) is modified to rapidly determine the nonlinear fluid film force (NFFF) of a TPJB while considering turbulent and thermal effects. A high-accuracy, large-capacity NFFF database for a single pad is constructed by numerically solving the turbulent adiabatic hydrodynamic model for five equivalent state variables of the journal, which are discretized in the pad coordinates. The remaining variables are not discretized in the DM. A combined linear and parabolic interpolation polynomial based on the database is established to accurately calculate the NFFF of the tilting pads; thus, the NFFF of a four-pad TPJB is obtained in the bearing coordinates. The DM is applied to analyze and compare the nonlinear dynamic behavior of a water-lubricated TPJB-Jeffcott rotor system with and without turbulent and thermal effects. The present DM solution without these effects and the previous DM solution are shown to be consistent. The results demonstrate the importance of the flow regime and the negligibility of temperature increases in the nonlinear dynamics of a water-lubricated TPJB. This work contributes to the accurate and efficient analysis of the nonlinear dynamics of high-speed TPJBs and low-viscosity-fluid-lubricated TPJBs.
\end{abstract}

Keywords: tilting-pad journal bearing; nonlinear fluid film force; rotor dynamics; turbulent flow; thermal effect

\section{Introduction}

Owing to good stability and adaptability, tilting-pad journal bearings (TPJBs) have been widely used in large rotating components, such as those in steam turbines, compressors, and nuclear reactor coolant pumps. Low-viscosity fluids, such as water, have been used as journal bearing lubricants in ship stern shafts, hydraulic turbines, and water pumps. In addition, water-lubricated TPJBs have been used in the nuclear reactor coolant pump of the third-generation AP1000 reactor. Because of the nonlinear effect of the fluid film force of a TPJB, the unstable behaviors of a TPJB-rotor system, such as subharmonic rotor resonance and pad fluttering, cannot be accurately predicted using a linear model based on stiffness and damping coefficients, even though the linear theory for TPJBs is basically complete. However, an increased workload and calculation time are to complete a dynamic analysis of a TPJB-rotor system using the nonlinear model represented by Reynolds equation solutions. Therefore, researchers are currently pursuing a rapid and accurate method for calculating the nonlinear fluid film force (NFFF) of a TPJB to enable a simple and rapid nonlinear dynamic analysis of TPJB-rotor systems.

Some researchers have used infinitely long and short journal bearing models to analyze the dynamic performance of a journal bearing-rotor system. Wang and Khonsari [1,2] presented an analytical NFFF expression for an axially grooved infinitely long journal

*Corresponding author: Xiaoyang YUAN, E-mail: zonghezu_xjtu@163.com 


\begin{tabular}{|c|c|c|}
\hline \multicolumn{3}{|c|}{ Nomenclature } \\
\hline$M_{\mathrm{j}}, M_{\mathrm{d}}$ & Dimensionless masses of the journal and disk & $\rho, c_{v}, \mu, T$ Fluid density, specific heat, dynamic \\
\hline$X, Y$ & Dimensionless Cartesian coordinates & viscosity, and temperature \\
\hline$X_{\mathrm{j}}, Y_{\mathrm{j}}$ & Dimensionless journal displacements & $K_{\varphi}, K_{\lambda}, \tau_{c}$ Turbulent flow coefficients \\
\hline$X_{\mathrm{d}}, Y_{\mathrm{d}}$ & Dimensionless disk displacements & $\varphi, \lambda \quad$ Dimensionless circumferential and axial \\
\hline$X_{j}^{\prime \prime}, Y_{j}^{\prime \prime}$ & Dimensionless journal accelerations & coordinates \\
\hline$X_{\mathrm{d}}{ }^{\prime \prime}, Y_{\mathrm{d}}^{\prime \prime}$ & Dimensionless disk accelerations & Preload factor \\
\hline$F X, F Y$ & Dimensionless NFFF of the TPJB & $\beta \quad$ Pivot position angle \\
\hline$F X_{i}, F Y_{i}$ & Dimensionless NFFF of the $i$-th pad & $\varepsilon, \theta, \varepsilon^{\prime}, \varepsilon \theta^{\prime}$ Eccentricity ratio, attitude angle, dimen- \\
\hline K & Dimensionless rotor stiffness & sionless radial \\
\hline G & Dimensionless gravitational acceleration & velocity of the journal \\
\hline$\varepsilon_{\mathrm{d}}$ & Unbalanced mass eccentricity ratio of the disk & $\bar{\varepsilon}, \bar{\theta}, \bar{\varepsilon}^{\prime}, \bar{\varepsilon} \bar{\theta}^{\prime} \quad$ Equivalent eccentricity ratio, attitude \\
\hline$\tau$ & Dimensionless time & angle, dimensionless radial velocity, and \\
\hline $\bar{M}_{i}, \bar{J}$ & $\begin{array}{l}\text { Dimensionless torque and moment of inertia } \\
\text { of the } i \text {-th pad }\end{array}$ & $\begin{array}{ll} & \text { tangential velocity of the journal } \\
\bar{\varepsilon}_{\max }(\bar{\theta}) \quad \begin{array}{l}\text { Maximum equivalent eccentricity ratio as a } \\
\text { function of } \bar{\theta}\end{array}\end{array}$ \\
\hline$K_{\delta}, C_{\delta}$ & Dimensionless stiffness and damping of the & Clearance ratio \\
\hline & spring-damper & Radial clearance \\
\hline$\delta_{i}, \delta_{i}^{\prime}, \delta_{i}^{\prime \prime}$ & Dimensionless tilting angle, tilting velocity, & Pad radius \\
\hline & and tilting acceleration of the $i$-th pad & $R e_{\mathrm{e}}, R e_{\mathrm{c}}, R e_{\mathrm{m}} \quad$ Effective Reynolds number, critical Rey- \\
\hline$\alpha$ & Pad arc angle & nolds number, and mean Reynolds number \\
\hline Pc & Pivot coefficient & Journal speed and angular speed \\
\hline$H$ & Dimensionless fluid film thickness & Dimensionless inlet dynamic viscosity and \\
\hline$P$ & Dimensionless fluid film pressure & mean dynamic viscosity \\
\hline $\begin{array}{l}D, L \\
\bar{\mu}, \bar{T}, \bar{T}_{0}\end{array}$ & $\begin{array}{l}\text { Pad diameter and length } \\
\text { Dimensionless dynamic viscosity, fluid }\end{array}$ & $\begin{array}{l}\text { Position angles at the inlet and outlet } \\
\text { boundaries of the fluid film }\end{array}$ \\
\hline & film temperature, and inlet temperature & $\rho_{\mathrm{p},} h_{\mathrm{p}} J \quad$ Pad density, thickness, and moment of inertia \\
\hline
\end{tabular}

bearing considering the inlet pressure and position and investigated the influence of inlet pressure and position on the nonlinear dynamic performance of the bearing-rotor system. Chang-Jian [3] analyzed the nonlinear dynamics of a gear pair system supported by journal bearings using a long bearing approximation. Avramov and Borysiuk [4] studied the nonlinear dynamics of an asymmetrical one-disk rotor-bearing system based on the short bearing theory, and Shi et al. [5] studied the nonlinear dynamic behavior of a vertical rotor-bearing system based on the short bearing theory. Dakel et al. [6] investigated the linear and nonlinear dynamic behavior of an on-board rotorbearing system using the short bearing model. Okabe and Cavalca [7] developed a short bearing model of a TPJB with turbulent effects and analyzed the dynamic behavior of a Jeffcott rotor-TPJB system with and without turbulent effects. Some researchers have reported an alytical models and calculation methods for the NFFF and pressure distribution and for nonlinear dynamic analysis of finite-length journal bearing-rotor systems. Zhang and Xu [8] proposed an NFFF model expressed by two symmetric positive definite matrices composed of three functions. Zhao et al. [9] investigated the linear and nonlinear unbalance response of a symmetrical single-disk flexible rotor-bearing system. The NFFF was obtained by solving the Reynolds equation and energy equation using the finite element method (FEM) at each time step. Xia et al. [10] provided two tools, a Ritz model and a one-dimensional FEM approach, for calculating the NFFF based on the free boundary theory and variational method under the 
Reynolds boundary condition, which demonstrated high efficiency. Bastani and de Queiroz [11] applied correction functions to force models of infinitely long and short bearings and proposed a closed-form NFFF expression. Vignolo et al. [12] treated the Ocvirk number as an expandable parameter and proposed an approximate analytical solution of the Reynolds equation using the regular perturbation method. Sfyris and Chasalevris [13] added a set of particular solutions for the Reynolds equation to a general solution of the homogenous Reynolds equation using the method of separation of variables, thus obtaining a closed-form expression of lubricant pressure. Zhang et al. [14] expressed the pressure distribution of an infinitely long journal bearing as a circumferential separable function of pressure distribution and obtained an axial separable function of pressure distribution through the variational principle, thus providing an approximate analytical method for calculating the NFFF of a turbulent journal bearing. Hei et al. [15] obtained the NFFF by the method of separation of variables based on the variational principle and investigated the nonlinear dynamic behavior of a rod-fastening rotorbearing system. Abu-Mahfouz and Adams [16] expanded the NFFF of a pad in a Taylor series, obtained the instantaneous linearized stiffness and damping coefficients, which were updated at each time step, and analyzed the nonlinear vibration of an unbalanced rotor supported by a three-pad TPJB under an on-pad load and a non-static biasing load. Wang et al. [17] established an NFFF model of a single pad through the method of separation of variables under the dynamic $\pi$ fluid film boundary condition, resulting in a NFFF model of a four-pad TPJB based on the pad assembly method.

In 2002, Chen et al. [18] proposed the database method (DM) to calculate the NFFF by introducing a speed parameter ranging from -1 to 1 to the manipulative Reynolds equation. The effectiveness of the $\mathrm{DM}$ was verified by analyzing the nonlinear dynamic characteristics of an elliptical bearing-rigid rotor system. The DM presents a new approach for rapidly and accurately analyzing the nonlinear dynamics of journal bearing-rotor systems. Qin et al. [19] constructed a NFFF database of hydrodynamic bearings based on neural networks and studied the nonlinear motion characteristics of an elliptical bearing-rigid rotor system. Jin et al. [20] analyzed the nonlinear dynamic behavior, critical speed, and threshold speed of a supercritical rotor-bearing system using the DM based on the Poincare transformation. Ying et al. [21] studied the nonlinear dynamic response of a Jeffcott rotor-TPJB system with and without pad inertial effects using the DM. Lü et al. [22] investigated the nonlinear dynamics of a Jeffcott rotor supported by fixed-tilting-pad journal bearings using the DM, and Hei et al. [23] analyzed the nonlinear dynamic behavior of a rod-fastening rotor supported by fixed-tilting-pad journal bearings based on the DM.

Based on the above research, the NFFF models and calculation methods can be categorized as follows: i) infinitely long and short bearing models; ii) analytical models of finite-length journal bearings; iii) numerical methods; iv) the DM. Because the DM is based on accurate numerical methods and interpolation methods, we expect that the DM can rapidly and accurately calculate the NFFF for finite-length journal bearings. However, the DM is generally applied with isothermal and laminar assumptions, which inevitably introduce errors in the calculation. These errors will increase for high-speed bearings and low-viscosity-fluid-lubricated bearings.

In this paper, turbulent and thermal effects are applied in the DM to rapidly and accurately calculate the NFFF of a TPJB. The nonlinear dynamic behaviors of a water-lubricated TPJB-Jeffcott rotor system with and without the turbulent and thermal effects are compared and analyzed using the DM. The primary novelty of this work is as follows: i) the previous DM is modified by considering turbulent and thermal effects, but these effects are not introduced as discretized variables in the present DM; ii) the database capacity is far larger, and the NFFF data in the database are more accurate than in the previous database; iii) a combined linear and parabolic interpolation polynomial based on the database is established to calculate the NFFF of a TPJB more accurately.

\section{Mathematical model of the TPJB-rotor system}

A four-pad TPJB-Jeffcott rotor system is taken as the research object, as shown in Fig. 1. A brake springdamper is set on the outlet edges of the third and fourth 

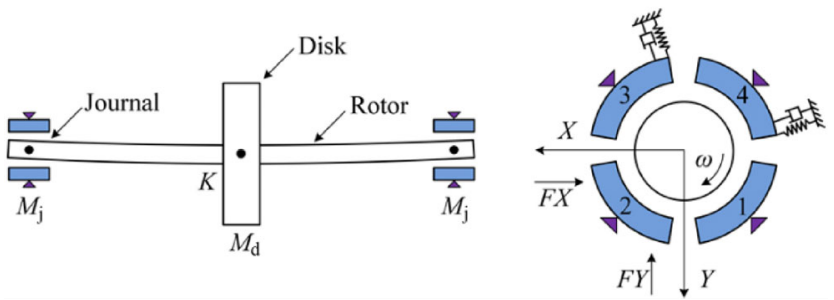

Fig. 1 Model of a Jeffcott rotor supported by two four-pad TPJBs.

pad backs of the TPJB to avoid fluttering of the two unloaded pads, including the rub impact between the pads and the journal. The dimensionless dynamic equations of the TPJB-rotor system can be expressed as follows:

$$
\left\{\begin{array}{l}
M_{\mathrm{j}} X_{\mathrm{j}}^{\prime \prime}=K\left(X_{\mathrm{d}}-X_{\mathrm{j}}\right)-F X \\
M_{\mathrm{j}} Y_{\mathrm{j}}^{\prime \prime}=K\left(Y_{\mathrm{d}}-Y_{\mathrm{j}}\right)+M_{\mathrm{j}} G-F Y \\
M_{\mathrm{d}} X_{\mathrm{d}}^{\prime \prime}=-2 K\left(X_{\mathrm{d}}-X_{\mathrm{j}}\right)+M_{\mathrm{d}} \varepsilon_{\mathrm{d}} \sin \tau \\
M_{\mathrm{d}} Y_{\mathrm{d}}^{\prime \prime}=-2 K\left(Y_{\mathrm{d}}-Y_{\mathrm{j}}\right)+M_{\mathrm{d}} G+M_{\mathrm{d}} \varepsilon_{\mathrm{d}} \cos \tau \\
\bar{J} \delta_{i}^{\prime \prime}=\bar{M}_{i}, i=1,2 \\
\bar{J} \delta_{i}^{\prime \prime}=\bar{M}_{i}-\frac{4 K_{\delta} \delta_{i}}{\psi}\{1-\cos [\alpha(1-\mathrm{Pc})]\} \\
\quad-\frac{4 C_{\delta} \delta_{i}^{\prime}}{\psi}\left\{1-\cos \left[\alpha\left(1-\mathrm{Pc}_{\mathrm{c}}\right)\right]\right\}, i=3,4
\end{array}\right.
$$

where $M_{\mathrm{j}}$ and $M_{\mathrm{d}}$ are the masses of the journal and disk, respectively; $\left(X_{j}, X_{d}, Y_{j}, Y_{d}\right)$ and $\left(X_{j}{ }^{\prime \prime}, X_{d}{ }^{\prime \prime}, Y_{j}{ }^{\prime \prime}, Y_{d}{ }^{\prime \prime}\right)$ are the displacements and accelerations, respectively, of the journal and disk in the $X$ and $Y$ directions; $F X$ and $F Y$ are the NFFF of the TPJB in the $X$ and $Y$ directions, respectively; $K$ is the rotor stiffness; $G$ is the gravitational acceleration; $\varepsilon_{\mathrm{d}}$ is the unbalanced mass eccentricity ratio of the disk; $\tau$ is a time variable; $\bar{M}_{i}$ and $\bar{J}$ are the torque and moment of inertia of the $i$-th pad, respectively; $K_{\delta}$ and $C_{\delta}$ are the stiffness and damping of the spring-damper, respectively; $\delta_{i}, \delta_{i}^{\prime}$, and $\delta_{i}{ }^{\prime \prime}$ are the tilting angle, tilting velocity, and tilting acceleration of the $i$-th pad, respectively; $\alpha$ is the pad arc angle; and $P c$ is the pivot coefficient. All parameters are dimensionless, and the dimensional ratios utilized in this paper are shown in the Appendix.

For the single pad shown in Fig. 2, the fluid film pressure distribution is governed by the Reynolds equation. The dimensionless dynamic Reynolds equation under incompressible turbulent flow conditions can

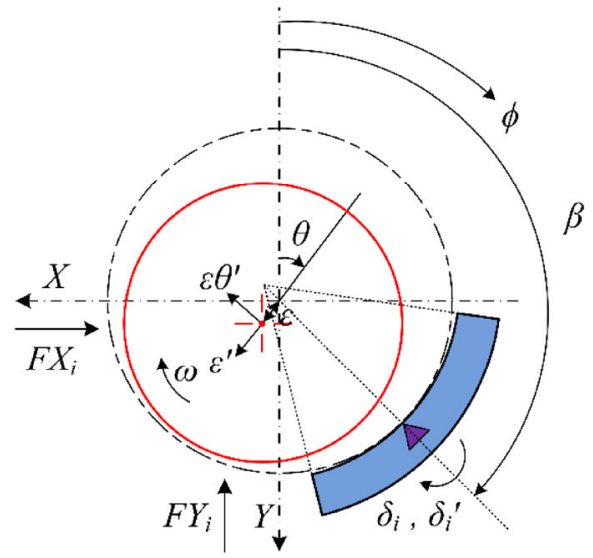

Fig. 2 Coordinate system of a single pad.

be written as follows:

$$
\frac{\partial}{\partial \phi}\left(\frac{H^{3}}{K_{\phi} \bar{\mu}} \frac{\partial P}{\partial \phi}\right)+\left(\frac{D}{L}\right)^{2} \frac{\partial}{\partial \lambda}\left(\frac{H^{3}}{K_{\lambda} \bar{\mu}} \frac{\partial P}{\partial \lambda}\right)=3 \frac{\partial H}{\partial \phi}+6 \frac{\partial H}{\partial \tau}
$$

where $H$ is the fluid film thickness; $P$ is the fluid film pressure; $D$ is the pad diameter; $L$ is the pad length; $\bar{\mu}$ is the dynamic viscosity; $K_{\varphi}$ and $K_{\lambda}$ are turbulent flow coefficients; and $\varphi$ and $\lambda$ are circumferential and axial coordinates, respectively.

The dimensionless fluid film thickness equation can be expressed as follows:

$$
H=1-A \cos (\beta-\phi)+\varepsilon \cos (\phi-\theta)+\frac{\delta_{i}}{\psi} \sin (\beta-\phi)
$$

where $A$ is a preload factor; $\beta$ is the pivot position angle; $\varepsilon$ is the journal eccentricity ratio; $\theta$ is the journal attitude angle; and $\psi=c / R$ is the clearance ratio, where $c$ is the radial clearance and $R$ is the pad radius.

Equation (3) can be transformed as an equivalent expression without $A$ and $\delta_{i}$ :

$$
H=1+\bar{\varepsilon} \cos (\phi-\bar{\theta})
$$

After substituting Eq. (4) into Eq. (2), Eq. (2) can be derived as follows:

$$
\begin{aligned}
& \frac{\partial}{\partial \phi}\left(\frac{H^{3}}{K_{\phi} \bar{\mu}} \frac{\partial P}{\partial \phi}\right)+\left(\frac{D}{L}\right)^{2} \frac{\partial}{\partial \lambda}\left(\frac{H^{3}}{K_{\lambda} \bar{\mu}} \frac{\partial P}{\partial \lambda}\right) \\
& =-3\left(\bar{\varepsilon}-2 \bar{\varepsilon} \bar{\theta}^{\prime}\right) \sin (\phi-\bar{\theta})+6 \bar{\varepsilon}^{\prime} \cos (\phi-\bar{\theta})
\end{aligned}
$$

where $\bar{\varepsilon}, \bar{\theta}, \bar{\varepsilon}^{\prime}$, and $\bar{\varepsilon} \bar{\theta}^{\prime}$ are the equivalent eccentricity ratio, attitude angle, radial velocity, and tangential 
velocity of the journal, respectively, as derived in the Appendix.

The fluid film temperature distribution is governed by the energy equation. The dimensionless adiabatic energy equation can be written as follows:

$$
\begin{aligned}
& \left(3 H-\frac{H^{3}}{K_{\phi} \bar{\mu}} \frac{\partial P}{\partial \phi}\right) \frac{\partial \bar{T}}{\partial \phi}-\left(\frac{D}{L}\right)^{2} \frac{H^{3}}{K_{\lambda} \bar{\mu}} \frac{\partial P}{\partial \lambda} \frac{\partial \bar{T}}{\partial \lambda} \\
& =\frac{6 \tau_{c} \bar{\mu}}{H}+\frac{2 H^{3}}{K_{\phi} \bar{\mu}}\left(\frac{\partial P}{\partial \phi}\right)^{2}+\left(\frac{D}{L}\right)^{2} \frac{2 H^{3}}{K_{\lambda} \bar{\mu}}\left(\frac{\partial P}{\partial \lambda}\right)^{2}
\end{aligned}
$$

where $\bar{T}$ is the fluid film temperature and $\tau_{\mathrm{c}}$ is the turbulent flow coefficient.

The Vogel viscosity-temperature relationship is adopted as follows:

$$
\mu=t_{1} \cdot \exp \left[t_{2} /\left(T+t_{3}\right)\right]
$$

where $\mu$ and $T$ are the dynamic viscosity and temperature of the fluid film, respectively, with dimensionless forms of $\bar{\mu}$ and $\bar{T}$. For a water film, $t_{1}, t_{2}$, and $t_{3}$ have been calculated as $2.5016 \times 10^{-5}, 569.21773$, and 134.17565, respectively, through a fitting method.

The appropriate values of $K_{\varphi}, K_{\lambda}$, and $\tau_{\mathrm{c}}$ are given by the following [24, 25]:

$$
\begin{gathered}
K_{\phi}=1+\frac{0.0136}{12} R e_{\mathrm{e}}^{0.90}, K_{\lambda}=1+\frac{0.0043}{12} R e_{\mathrm{e}}^{0.96}, \\
\tau_{\mathrm{c}}=1+0.0012 R e_{\mathrm{e}}^{0.94}
\end{gathered}
$$

where $R e_{\mathrm{e}}$ is the effective Reynolds number, which can be expressed for three types of flow regime conditions determined by the critical Reynolds number $R e_{c}$ including the laminar flow regime, transitional flow regime, and turbulent flow regime. $R e_{\mathrm{e}}$ can be expressed as follows:

$$
R e_{\mathrm{e}}=\left\{\begin{array}{lll}
0, & R e_{\mathrm{m}}<R e_{\mathrm{c}}, & \text { laminar } \\
\left(\frac{R e_{\mathrm{m}}}{R e_{\mathrm{c}}}-1\right) \frac{\rho \omega R H c}{\mu}, & R e_{\mathrm{c}} \leq R e_{\mathrm{m}} \leq 2 R e_{\mathrm{c}}, & \text { transitional } \\
\frac{\rho \omega R H c}{\mu}, & R e_{\mathrm{m}}>2 R e_{\mathrm{c}}, & \text { turbulent }
\end{array}\right.
$$

where $\rho$ is the fluid density; $\omega$ is the journal angular speed; $R e_{\mathrm{c}}=41.2 \sqrt{R / c}$; and $R e_{\mathrm{m}}=\rho \omega R c / \mu_{\mathrm{m}}$ is the mean Reynolds number. $\mu_{\mathrm{m}}$ is the mean dynamic viscosity, expressed as follows:

$$
\mu_{\mathrm{m}}=\frac{\mu_{0}}{2 \alpha} \int_{-1}^{1} \int_{\phi_{1}}^{\phi_{2}} \bar{\mu} \mathrm{d} \phi \mathrm{d} \lambda
$$

where $\mu_{0}$ is the inlet dynamic viscosity and $\varphi_{1}$ and $\varphi_{2}$ are the position angles at the inlet and outlet boundaries of the fluid film, respectively.

The dimensionless NFFF and torque of the pad can be written as follows:

$$
\begin{gathered}
\left\{\begin{array}{l}
F X_{i}=-\int_{-1}^{1} \int_{\phi_{1}}^{\phi_{2}} P \sin \phi \mathrm{d} \phi \mathrm{d} \lambda \\
F Y_{i}=-\int_{-1}^{1} \int_{\phi_{1}}^{\phi_{2}} P \cos \phi \mathrm{d} \phi \mathrm{d} \lambda
\end{array}\right. \\
\bar{M}_{i}=\int_{-1}^{1} \int_{\phi_{1}}^{\phi_{2}} P \sin (\beta-\phi) \mathrm{d} \phi \mathrm{d} \lambda
\end{gathered}
$$

Then, the dimensionless NFFF of the four-pad TPJB is

$$
F X=\sum_{i=1}^{4} F X_{i}, F Y=\sum_{i=1}^{4} F Y_{i}
$$

\section{Boundary conditions and computation}

For the pressure field, the classical Reynolds boundary conditions are adopted for Eq. (5):

$$
\begin{gathered}
P\left(\phi_{1}, \lambda\right)=0, P\left(\phi_{2}, \lambda\right)=0, \partial P(\phi, \lambda) /\left.\partial \phi\right|_{\phi=\phi_{2}}=0, \\
P(\phi, \pm 1)=0
\end{gathered}
$$

For the temperature field, the fluid film temperature at the inlet boundary is usually set as the inlet temperature $\bar{T}_{0}$, and heat is assumed to be completely transmitted by the pad under reverse flow conditions. Thus, the boundary conditions for Eq. (6) are given as follows:

$$
\bar{T}\left(\phi_{1}, \lambda\right)=\bar{T}
$$

if in the reverse flow area, then let $\bar{T}=\bar{T}_{0}$.

The dynamic Reynolds equation, Eq. (5), and energy equation, Eq. (6), are solved using the finite difference method (FDM) with successive over-relaxation iterations. The entire fluid film area is divided into $21 \times 17$ grid nodes in the $\varphi$ and $\lambda$ directions, respectively. The central difference scheme is applied to the dynamic Reynolds equation, and the upwind difference scheme 
is applied to the energy equation. Relative iterative convergence for the pressure and temperature is achieved within $10^{-5}$ and $10^{-4}$, respectively.

\section{Database method and NFFF interpolation polynomial}

Through the treatment of Eq. (4), Eq. (2) is transformed into Eq. (5). The eccentricity ratio $\varepsilon$, attitude angle $\theta$, and pad tilting angle $\delta_{i}$ are replaced by two new variables, the equivalent eccentricity ratio $\bar{\varepsilon}$ and the equivalent attitude angle $\bar{\theta}$, with value ranges of $\left[0, \bar{\varepsilon}_{\max }(\bar{\theta})\right]$ and $[0,2 \pi]$, respectively. The derivation of $\bar{\varepsilon}_{\max }(\bar{\theta})$ is given in the Appendix. The radial velocity $\varepsilon^{\prime}$, tangential velocity $\varepsilon \theta^{\prime}$, and pad tilting velocity $\delta_{i}^{\prime}$ are replaced by another two new variables, the equivalent radial velocity $\vec{\varepsilon}^{\prime}$ and the equivalent tangential velocity $\bar{\varepsilon} \bar{\theta}^{\prime}$, which have wider theoretical value ranges. Here, consideration of the value ranges of $\bar{\varepsilon}, \bar{\varepsilon} \bar{\theta}^{\prime}$, and the journal speed $n$ is optional. Thus far, the seven state variables $\left(\varepsilon, \theta, \varepsilon^{\prime}, \varepsilon \theta^{\prime}, \delta_{i}, \delta_{i}^{\prime}, n\right)$ representing the motion states of the journal and the $i$-th pad have been integrated into only five equivalent state variables $\left(\bar{\varepsilon}, \bar{\theta}, \bar{\varepsilon}, \bar{\varepsilon} \bar{\theta}^{\prime}, n\right)$. After properly discretizing the five equivalent state variables within their respective value ranges, the NFFF database can be constructed by storing all sets of $\left(\bar{\varepsilon}, \bar{\theta}, \bar{\varepsilon}^{\prime}, \bar{\varepsilon} \bar{\theta}^{\prime}\right.$, $n$ ) and the corresponding calculated NFFF values in a text file. The process for constructing the NFFF database is shown in Fig. 3.

For an arbitrary set of seven state variables $\left(\varepsilon, \theta, \varepsilon^{\prime}\right.$, $\left.\varepsilon \theta^{\prime}, \delta_{i}, \delta_{i}^{\prime}, n\right)$, the corresponding five equivalent state variables $\left(\bar{\varepsilon}, \bar{\theta}, \bar{\varepsilon}^{\prime}, \bar{\varepsilon} \bar{\theta}^{\prime}, n\right)$ can be calculated using Eqs. (A1), (A2), (A3), and (A4); then, the corresponding $\operatorname{NFFF}\left(F X_{i}, F Y_{i}\right)$ can be identified in the database. If the five calculated state variables, e.g., $\left(\bar{\varepsilon}_{i}, \bar{\theta}_{i}, \bar{\varepsilon}_{i}^{\prime}, \bar{\varepsilon} \bar{\theta}_{i}^{\prime}\right.$, $\left.n_{i}\right)$, are not included in the database, the NFFF will be evaluated by an interpolation method using the existing NFFF at the nodes closest to $\left(\bar{\varepsilon}_{i}, \bar{\theta}_{i}, \bar{\varepsilon}_{i}^{\prime}, \bar{\varepsilon} \bar{\theta}_{i}^{\prime}, n_{i}\right)$. The piecewise linear interpolation method for $\left(\bar{\varepsilon}_{i}^{\prime}, \bar{\varepsilon} \bar{\theta}_{i}^{\prime}, n_{i}\right)$ and the piecewise quadratic interpolation method for $\left(\bar{\varepsilon}_{i}, \bar{\theta}_{i}\right)$ are used to construct an interpolation polynomial of $\mathrm{FX}_{i}$ and $\mathrm{FY}_{i}$, given as follows:

$$
F X_{i}=S V \cdot C F X, F Y_{i}=S V \cdot C F Y
$$

where

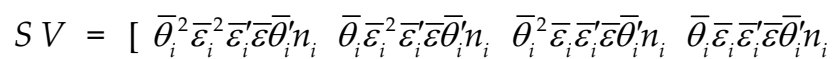
$\bar{\theta}_{i} \bar{\varepsilon}_{i} \bar{\varepsilon}_{i}^{\prime} \bar{\varepsilon} \bar{\theta}_{i}^{\prime} \bar{\theta}_{i}^{2} \bar{\varepsilon}_{i} \bar{\varepsilon}_{i} \bar{\varepsilon} \bar{\theta}_{i}^{\prime} \quad \bar{\theta}_{i} \bar{\varepsilon}_{i}^{2} \bar{\varepsilon}_{i}^{\prime} \overline{\theta_{i}^{\prime}} \bar{\theta}_{i}^{2} \bar{\varepsilon}_{i}^{2} \bar{\varepsilon}_{i}^{\prime} \bar{\varepsilon} \bar{\theta}_{i}^{\prime} \bar{\theta}_{i} \bar{\varepsilon}_{i} \bar{\varepsilon}_{i}^{\prime} n_{i} \bar{\theta}_{i}^{2} \bar{\varepsilon}_{i} \bar{\varepsilon}_{i} n_{i}$ $\bar{\theta}_{i} \bar{\varepsilon}_{i}^{2} \bar{\varepsilon}_{i}^{\prime} n_{i} \bar{\theta}_{i}^{2} \bar{\varepsilon}_{i}^{2} \bar{\varepsilon}_{i}^{\prime} n_{i} \bar{\theta}_{i} \bar{\varepsilon}_{i} \bar{\varepsilon} \bar{\theta}_{i}^{\prime} n_{i} \bar{\theta}_{i}^{2} \bar{\varepsilon}_{i} \bar{\varepsilon} \bar{\theta}_{i}^{\prime} n_{i} \bar{\theta}_{i} \bar{\varepsilon}_{i}^{2} \bar{\varepsilon} \bar{\theta}_{i}^{\prime} n_{i} \bar{\theta}_{i}^{2} \bar{\varepsilon}_{i}^{2} \bar{\varepsilon}_{i}^{\prime} n_{i}$ $\bar{\theta}_{i} \bar{\varepsilon}_{i}^{\prime} \bar{\varepsilon} \bar{\theta}_{i}^{\prime} n_{i} \quad \bar{\theta}_{i}^{2} \bar{\varepsilon}_{i}^{\prime} \bar{\varepsilon} \bar{\theta}_{i}^{\prime} n_{i} \quad \bar{\varepsilon}_{i} \bar{\varepsilon}_{i}^{\prime} \bar{\varepsilon} \bar{\theta}_{i}^{\prime} n_{i} \quad \bar{\varepsilon}_{i}^{2} \bar{\varepsilon}_{i}^{\prime} \bar{\varepsilon} \bar{\theta}_{i}^{\prime} n_{i} \quad \bar{\varepsilon}_{i}^{\prime} \bar{\varepsilon} \bar{\varepsilon}_{i}^{\prime} n_{i} \quad \bar{\varepsilon}_{i} \bar{\varepsilon} \bar{\theta}_{i}^{\prime} n_{i}$ $\bar{\varepsilon}_{i}^{2} \bar{\varepsilon} \bar{\theta}_{i}^{\prime} n_{i} \quad \bar{\varepsilon}_{i} \bar{\varepsilon}_{i}^{\prime} n_{i} \quad \bar{\varepsilon}_{i}^{2} \bar{\varepsilon}_{i}^{\prime} n_{i} \quad \bar{\varepsilon}_{i} \bar{\varepsilon}_{i}^{\prime} \bar{\varepsilon}_{i}^{\prime} \quad \bar{\varepsilon}_{i}^{2} \bar{\varepsilon}_{i}^{\prime} \overline{\varepsilon_{i}^{\prime}} \quad \bar{\theta}_{i} \bar{\varepsilon}_{i}^{\prime} n_{i} \quad \bar{\theta}_{i}^{2} \bar{\varepsilon}_{i}^{\prime} n_{i}$ $\bar{\theta}_{i} \bar{\varepsilon}_{i}^{\prime} n_{i} \bar{\theta}_{i}^{2} \bar{\varepsilon}_{i}^{\prime} n_{i} \bar{\theta}_{i} \bar{\varepsilon}_{i}^{\prime} \bar{\varepsilon} \bar{\theta}_{i}^{\prime} \bar{\theta}_{i}^{2} \bar{\varepsilon}_{i}^{\prime} \bar{\varepsilon}_{i}^{\prime} \quad \bar{\theta}_{i} \bar{\varepsilon}_{i} n_{i} \bar{\theta}_{i}^{2} \bar{\varepsilon}_{i} n_{i} \bar{\theta}_{i} \bar{\varepsilon}_{i}^{2} n_{i} \bar{\theta}_{i}^{2} \bar{\varepsilon}_{i}^{2} n_{i}$ $\bar{\theta}_{i} \bar{\varepsilon}_{i} \bar{\varepsilon} \bar{\theta}_{i}^{\prime} \quad \bar{\theta}_{i}^{2} \bar{\varepsilon}_{i} \bar{\varepsilon} \bar{\theta}_{i}^{\prime} \quad \bar{\theta}_{i} \bar{\varepsilon}_{i}^{2} \bar{\varepsilon} \bar{\theta}_{i}^{\prime} \quad \bar{\theta}_{i}^{2} \bar{\varepsilon}_{i}^{2} \bar{\varepsilon} \bar{\theta}_{i}^{\prime} \quad \bar{\theta}_{i} \bar{\varepsilon}_{i} \bar{\varepsilon}_{i}^{\prime} \quad \bar{\theta}_{i}^{2} \bar{\varepsilon}_{i} \bar{\varepsilon}_{i}^{\prime} \quad \bar{\theta}_{i} \bar{\varepsilon}_{i}^{2} \bar{\varepsilon}_{i}^{\prime}$ $\bar{\theta}_{i}^{2} \bar{\varepsilon}_{i}^{2} \bar{\varepsilon}_{i}^{\prime} \bar{\theta}_{i} \bar{\varepsilon}_{i} \quad \bar{\theta}_{i}^{2} \bar{\varepsilon}_{i} \bar{\theta}_{i} \bar{\varepsilon}_{i}^{2} \bar{\theta}_{i}^{2} \bar{\varepsilon}_{i}^{2} \bar{\theta}_{i} \bar{\varepsilon}_{i}^{\prime} \bar{\theta}_{i}^{2} \bar{\varepsilon}_{i}^{\prime} \bar{\theta}_{i} \bar{\varepsilon}_{\bar{\theta}}^{\prime} \bar{\theta}_{i}^{2} \bar{\varepsilon} \bar{\theta}_{i}^{\prime} \bar{\theta}_{i} n_{i}$ $\bar{\theta}_{i}^{2} n_{i} \bar{\varepsilon}_{i} \bar{\varepsilon}_{i}^{\prime} \quad \bar{\varepsilon}_{i}^{2} \bar{\varepsilon}_{i}^{\prime} \quad \bar{\varepsilon}_{i} \bar{\varepsilon} \bar{\theta}_{i}^{\prime} \quad \bar{\varepsilon}_{i}^{2} \bar{\varepsilon} \bar{\theta}_{i}^{\prime} \quad \bar{\varepsilon}_{i} n_{i} \bar{\varepsilon}_{i}^{2} n_{i} \bar{\varepsilon}_{i}^{\prime} \bar{\varepsilon} \bar{\theta}_{i}^{\prime} \quad \bar{\varepsilon}_{i}^{\prime} n_{i} \bar{\varepsilon} \bar{\theta}_{i}^{\prime} n_{i} \bar{\theta}_{i}$ $\left.\bar{\theta}_{i}^{2} \bar{\varepsilon}_{i} \bar{\varepsilon}_{i}^{2} \bar{\varepsilon}_{i}^{\prime} \bar{\varepsilon} \bar{\theta}_{i}^{\prime} n_{i} 1\right]$

$$
\begin{aligned}
& C F X=\left[\begin{array}{llll}
c f x 1 & c f x 2 & \cdots & c f x 72
\end{array}\right]^{\mathrm{T}}, \\
& C F Y=\left[\begin{array}{llll}
c f y 1 & c f y 2 & \cdots & c f y 72
\end{array}\right]^{\mathrm{T}}
\end{aligned}
$$

where $c f x 1-72$ and $c f y 1-72$ are the polynomial coefficients of $\mathrm{FX}_{i}$ and $\mathrm{FY}_{i}$, respectively, which can be calculated from the database using the NFFF at the nodes closest to $\left(\bar{\varepsilon}_{i}, \bar{\theta}_{i}, \bar{\varepsilon}_{i}^{\prime}, \bar{\varepsilon} \bar{\theta}_{i}^{\prime}, n_{i}\right)$. The NFFF of the other pads can also be conveniently obtained from the database using a coordinate transformation of the

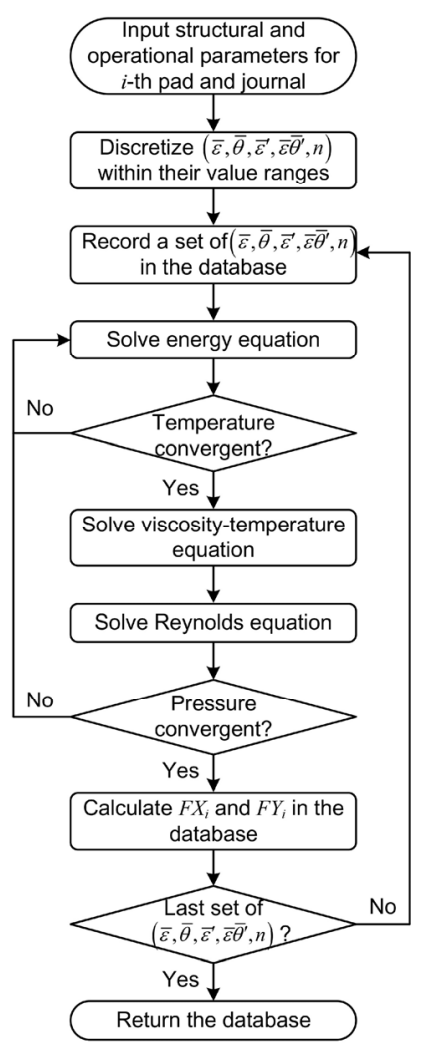

Fig. 3 Flow chart of the NFFF database construction. 
journal attitude angle. Equation (16) is a combined linear and parabolic interpolation polynomial with five equivalent state variables, and the NFFF can be rapidly calculated using an analytical expression based on the database instead of using numerical iterative methods. The process for accessing the NFFF database is shown in Fig. 4.

When constructing the NFFF database, the optional value ranges of $\bar{\varepsilon}^{\prime}, \bar{\varepsilon} \bar{\theta}^{\prime}$, and $n$ are tentatively taken as $[-0.3,0.3],[-0.3,0.3]$, and $[1000,9000]$, respectively. After numerous calculation result comparisons, it is found that when $\bar{\theta}, \bar{\varepsilon}^{\prime}, \bar{\varepsilon} \bar{\theta}^{\prime}$, and $n$ are discretized into $31,11,11$, and 11 nodes with equal intervals, respectively, and $\bar{\varepsilon}$ is discretized into 25 nodes with unequal intervals -4 equal intervals in $\left[0, \bar{\varepsilon}_{\max }(\bar{\theta}) / 3\right]$, 8 equal intervals in $\left[\bar{\varepsilon}_{\max }(\bar{\theta}) / 3,2 \bar{\varepsilon}_{\max }(\bar{\theta}) / 3\right]$, and 12 equal intervals in $\left[2 \bar{\varepsilon}_{\max }(\bar{\theta}) / 3, \bar{\varepsilon}_{\max }(\bar{\theta})\right]$, the DM has a suitable calculation precision and speed. Thus, these intervals are used to predict the axis orbits, dynamic fluid temperature, and pad motion of the TPJB-rotor system.

\section{Numerical results and discussion}

A precise time-integration method is adopted to solve Eq. (1). In this study, the time step for direct

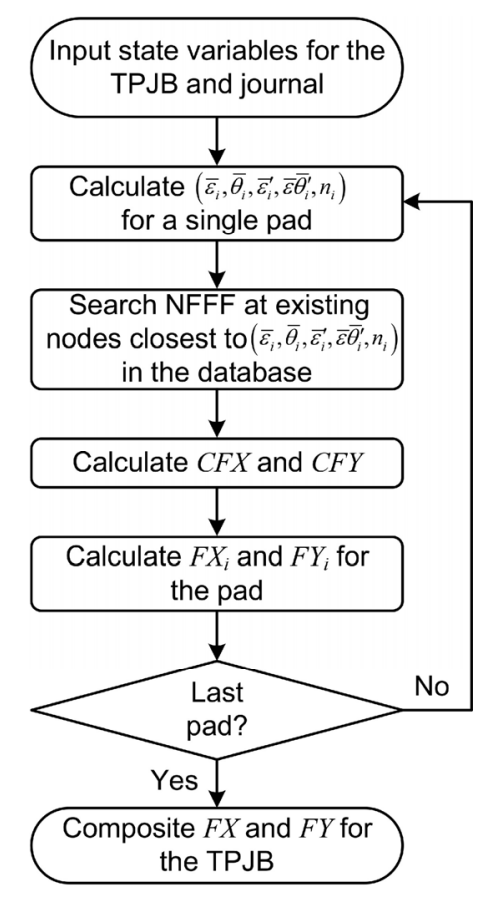

Fig. 4 Flow chart for accessing the NFFF database. numerical integration is specified as $\pi / 2500$, and the computational data under the steady state condition are extracted and analyzed. The calculation is performed by MATLAB using an Intel Core i7-5500U at $2.40 \mathrm{GHz}$. The structural and operational parameters of the TPJB-rotor system are listed in Table 1, and the expression of the pad moment of inertia is given in the Appendix. Because water has been employed in the nuclear reactor coolant pump of the third-generation AP1000 reactor, water is chosen as the TPJB lubricant to study the effects of turbulence and temperature.

\subsection{Comparison of present DM and previous DM [18]}

In this section, the research object is a single pad of the water-lubricated TPJB, whose pivot position angle is $180^{\circ}$. A basic comparison of the present DM and the previous DM is presented in Table 2. It is observed that the present DM, which considers turbulent and thermal effects, has a very large database capacity and a large construction cost. Figure 5 compares the dimensionless NFFF calculated by the present DM and those obtained by the FDM and the previous DM. The solution obtained from the present DM with turbulent and thermal effects is in good agreement

Table 1 Structural and operational parameters of the TPJB-rotor system.

\begin{tabular}{ll}
\hline \multicolumn{1}{c}{ Parameter } & \multicolumn{1}{c}{ Value } \\
\hline Pad radius & $104 \mathrm{~mm}$ \\
Pad length & $195.5 \mathrm{~mm}$ \\
Preload factor & 0 \\
Pivot coefficient & 0.5 \\
Pad arc angle & $80^{\circ}$ \\
Clearance ratio & 0.000928 \\
Pad moment of inertia & $0.003 \mathrm{~kg} \cdot \mathrm{m}^{2}$ \\
Spring-damper stiffness & $1 \times 10^{5} \mathrm{~N} / \mathrm{m}$ \\
Spring-damper damping & $1 \times 10^{3} \mathrm{~N} \cdot \mathrm{s} / \mathrm{m}$ \\
Inlet fluid temperature & $30{ }^{\circ} \mathrm{C}$ \\
Fluid density & $995.7 \mathrm{~kg} \cdot \mathrm{m}^{-3}$ \\
Fluid specific heat & $4174 \mathrm{~J} /\left(\mathrm{kg} \cdot{ }^{\circ} \mathrm{C}\right)$ \\
Journal mass & $50 \mathrm{~kg}$ \\
Disk mass & $300 \mathrm{~kg}$ \\
Unbalanced mass eccentricity ratio & 0.2 \\
Rotor stiffness & $2.5 \times 10^{8} \mathrm{~N} / \mathrm{m}$ \\
\hline
\end{tabular}


Table 2 Basic comparison of the present DM and the previous DM.

\begin{tabular}{cccc}
\hline & $\begin{array}{c}\text { Present DM } \\
\text { (Turbulent and thermal) }\end{array}$ & $\begin{array}{c}\text { Present DM } \\
\text { (Laminar and isothermal) }\end{array}$ & Previous DM \\
\hline FDM grid & $20(\varphi) \times 16(\lambda)$ & $20(\varphi) \times 16(\lambda)$ & $20(\varphi) \times 16(\lambda)$ \\
Variables to be discretized & $\bar{\varepsilon}, \bar{\theta}, \bar{\varepsilon}, \bar{\varepsilon} \bar{\theta}^{\prime}, n$ & $\bar{\varepsilon}, \bar{\theta}, \bar{\varepsilon}, \bar{\varepsilon} \overline{\theta^{\prime}}$ & $(25,40,20)$ \\
(node number) & $(25,31,11,11,11)$ & $(25,31,11,11)$ & Equal \\
Node interval & Unequal for $\bar{\varepsilon}$ & Unequal for $\bar{\varepsilon}$ & Linear \\
Interpolation method & Quadratic and linear combination & Quadratic and linear combination & $2.43 \mathrm{M}$ \\
Database capacity & $79.9 \mathrm{M}$ & $6.24 \mathrm{M}$ & 5 min \\
Database construction time & $9.6 \mathrm{~h}$ & 5.6 min & \\
\hline
\end{tabular}

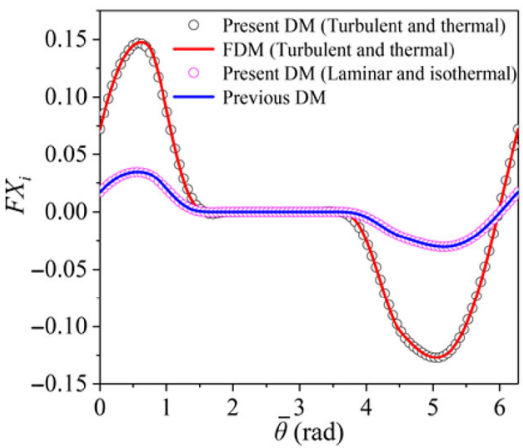

(a) $\bar{\varepsilon}=0.2, \vec{\varepsilon}=\bar{\varepsilon} \bar{\theta}^{\prime}=0.25, n=8000 \mathrm{rpm}$

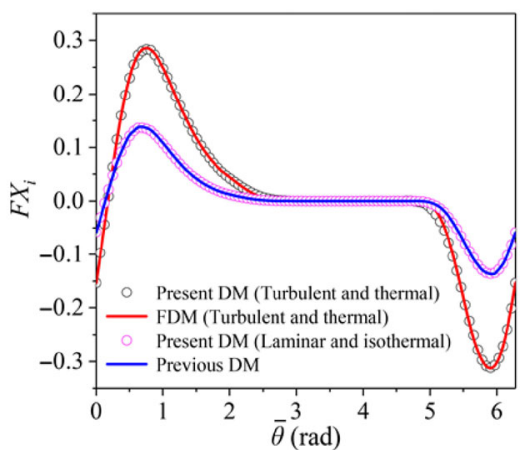

(c) $\bar{\varepsilon}=0.55, \bar{\varepsilon}=\bar{\varepsilon} \bar{\theta}^{\prime}=0.15, n=5000 \mathrm{rpm}$

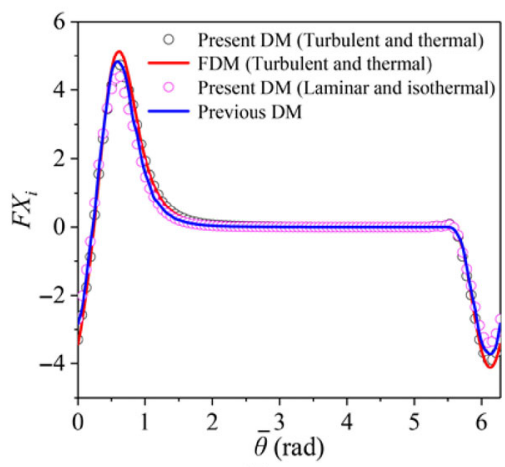

(e) $\bar{\varepsilon}=0.9, \vec{\varepsilon}=\bar{\varepsilon} \bar{\theta}^{\prime}=0.05, n=2000 \mathrm{rpm}$

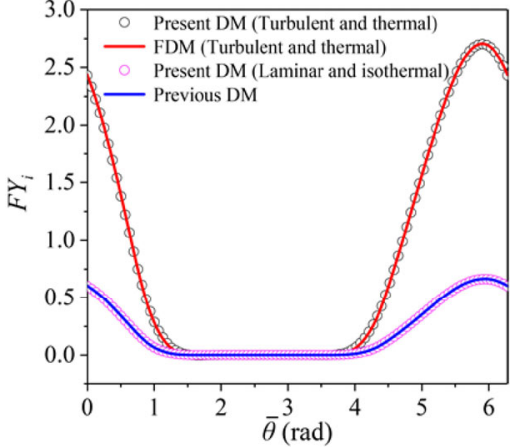

(b) $\bar{\varepsilon}=0.2, \vec{\varepsilon}=\bar{\varepsilon} \bar{\theta}^{\prime}=0.25, n=8000 \mathrm{rpm}$

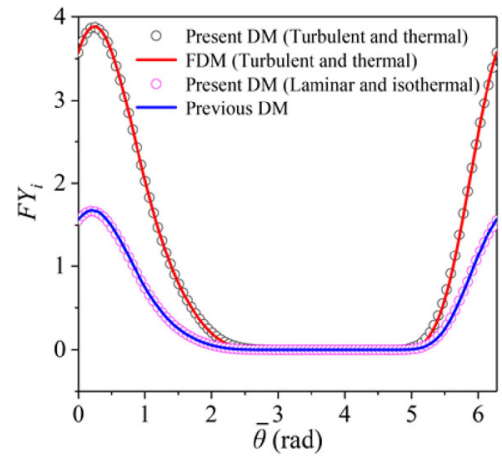

(d) $\bar{\varepsilon}=0.55, \vec{\varepsilon}=\bar{\varepsilon} \bar{\theta}^{\prime}=0.15, n=5000 \mathrm{rpm}$

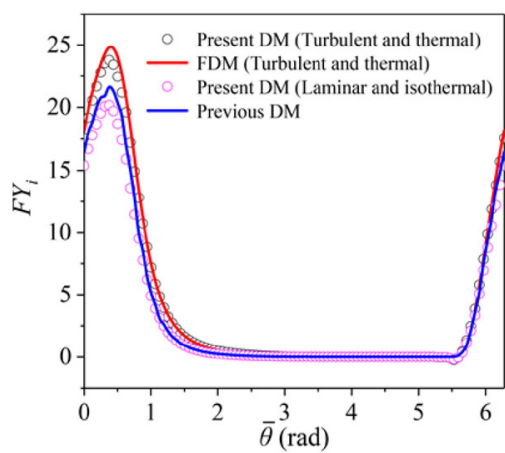

(f) $\bar{\varepsilon}=0.9, \vec{\varepsilon}=\bar{\varepsilon} \bar{\theta}^{\prime}=0.05, n=2000 \mathrm{rpm}$

Fig. 5 Present DM, FDM, and previous DM solutions for the dimensionless NFFF as a function of $\bar{\theta}$.

with the FDM solution with the same effects. However, the values are larger than those for the previous DM solution in some regions because the previous DM is based on isothermal and laminar assumptions.
Moreover, the present DM solution without turbulent or thermal effects is in good agreement with the previous DM solution, demonstrating that the constructed database is suitable. Figure 6 presents ten cycles of 


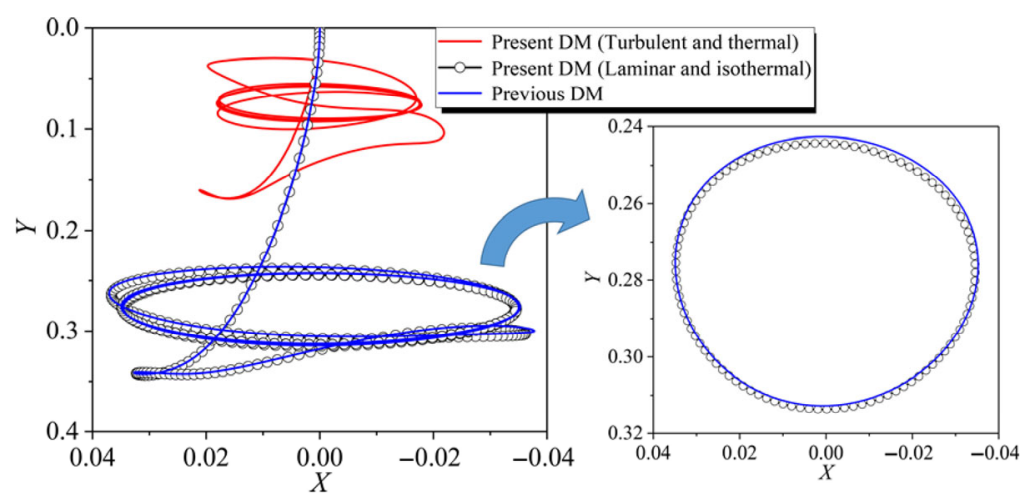

Fig. 6 Present DM and previous DM solutions for ten cycles of transient journal center orbits at 3,000 rpm.

transient journal center orbits calculated by the present $\mathrm{DM}$ and those determined by the previous DM at $3,000 \mathrm{rpm}$. The present DM solution without turbulent or thermal effects is in good agreement with the previous DM solution, while the present DM solution with these effects is closer to the bearing center and has a smaller orbit amplitude than the previous DM solution. The calculation costs for ten cycles of transient journal center orbits for the present DM and previous $\mathrm{DM}$ are shown in Table 3. It is observed that the calculation cost for the present DM with turbulent and thermal effects is approximately nine times larger than that with the previous DM; however, this cost is acceptable for performing nonlinear dynamic analysis.

\subsection{Synchronous rotor motion with and without turbulent and thermal effects}

Figure 7 shows the journal center orbits with and without turbulent and thermal effects. From Fig. 7, it can be seen that the orbit centers are approximately located at the $Y$ axis of the TPJB and move closer to the TPJB center as the speed increases. Moreover, the journal orbit amplitude increases as the speed increases. The journal orbit amplitudes with turbulent and thermal effects are smaller than those without these effects, except at 1,000 rpm. At 6,000 rpm, the journal orbit amplitude with turbulent and thermal effects is nearly three times smaller than the orbits without these effects. The orbits at 2,000 rpm-6,000 rpm with turbulent and thermal effects are much closer to the TPJB center than those without these effects.

Figure 8 shows the disk center orbits with and without turbulent and thermal effects. From Fig. 8, it can be seen that the trends for the disk center orbits are similar to those for the journal center orbits. However, the disk orbit amplitudes are larger than

Table 3 Calculation time for ten cycles of transient journal center orbits at 3,000 rpm with the present DM and previous DM.

\begin{tabular}{cccc}
\hline Present DM (Turbulent and thermal) & Present DM (Laminar and isothermal) & Previous DM \\
\hline $76 \mathrm{~s}$ & $21 \mathrm{~s}$ & $8 \mathrm{~s}$ \\
\hline
\end{tabular}

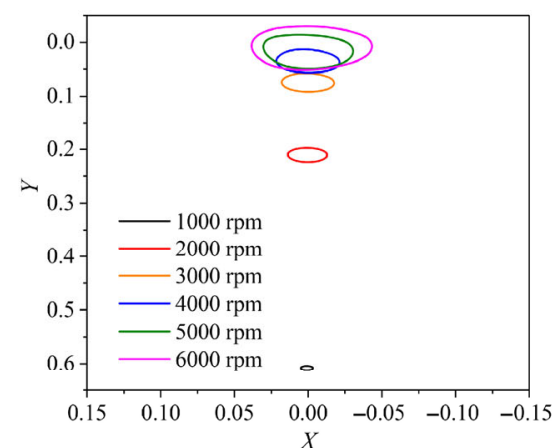

(a)

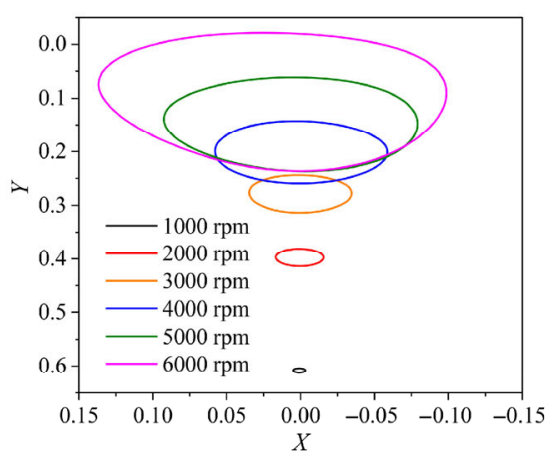

(b)

Fig. 7 Journal center orbits (a) with and (b) without turbulent and thermal effects. 


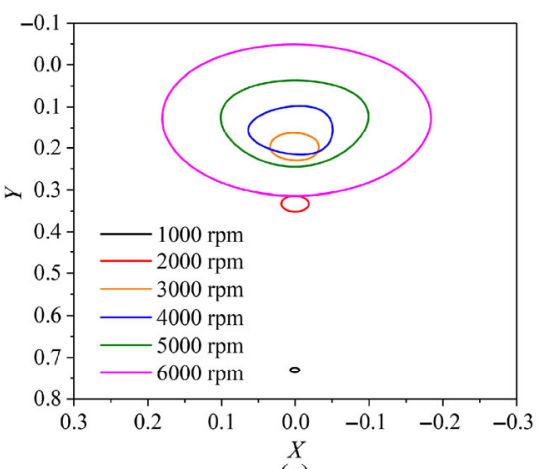

(a)

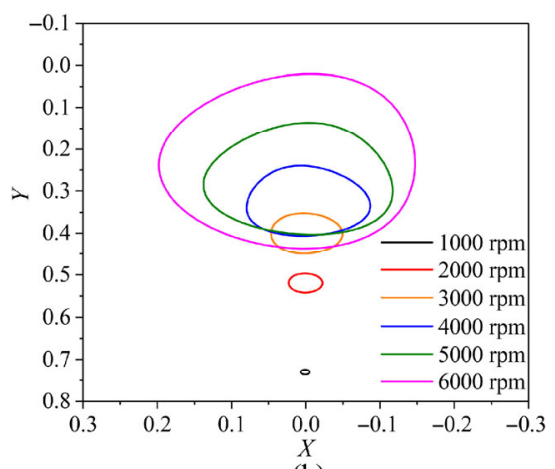

(b)

Fig. 8 Disk center orbits (a) with and (b) without turbulent and thermal effects.

the journal due to the rotor flexibility. The disk orbit amplitudes with turbulent and thermal effects are slightly smaller than those without these effects.

The temperature field of the fluid film on the first pad at 1,000 rpm and 6,000 rpm without an unbalanced mass is shown in Fig. 9. It can be seen that the fluid film temperature is almost constant in the $\lambda$ direction and increases almost linearly with increasing $\varphi$. The temperature increases by $2.5^{\circ} \mathrm{C}$ at $6,000 \mathrm{rpm}$ and by only $0.2^{\circ} \mathrm{C}$ at $1,000 \mathrm{rpm}$. The temperature rise of the fluid film for the water-lubricated TPJB-rotor system at $1,000 \mathrm{rpm}-6,000 \mathrm{rpm}$ is too small $\left(0.2^{\circ} \mathrm{C}-2.5^{\circ} \mathrm{C}\right)$ to have a substantial effect; thus, only the flow regime plays an important role in the dynamic analysis. For $1,000 \mathrm{rpm}, \operatorname{Re}_{\mathrm{e}}=0$ and $K_{\varphi}=K_{\lambda}=\tau_{\mathrm{c}}=1$, which correspond to laminar flow conditions; thus, the same axis orbit is obtained regardless of whether turbulent and thermal effects are considered. For 2,000 rpm-6,000 rpm, $K_{\varphi}>1$, $K_{\lambda}>1$, and $\tau_{c}>1$, indicating that the TPJB-rotor system is in a transitional flow or even the turbulent flow

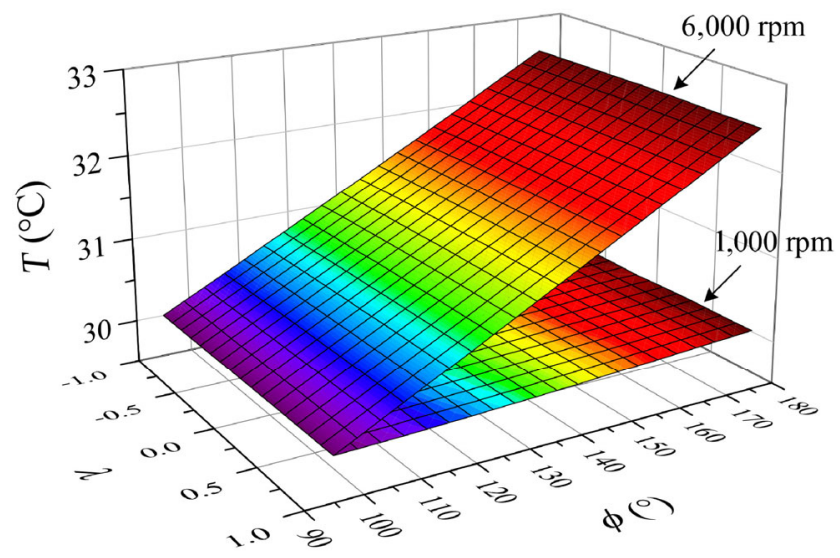

Fig. 9 Temperature field of the fluid film on the first pad at $1,000 \mathrm{rpm}$ and 6,000 rpm without an unbalanced mass. regime, which embodies in a higher dynamic viscosity. The increase in fluid viscosity will lead to an increased NFFF; thus, the calculated orbits are closer to the bearing center.

\subsection{Nonlinear journal behavior with and without turbulent and thermal effects}

Figures 10 and 11 show the journal center orbits and Poincaré maps for six representative speeds with and without turbulent and thermal effects, respectively. At these speeds, the orbits have distinct nonlinear characteristics, and a substantial difference is observed between the nonlinear characteristics with and without turbulent and thermal effects. At 6,500 rpm and $6,800 \mathrm{rpm}$, the journal motion is synchronous when turbulent and thermal effects are considered (see Figs. 10.1 and 10.2), while the journal exhibits period-4 motion and period-3 motion, respectively, when turbulent and thermal effects are neglected (see Figs. 11.1 and 11.2). At 7,600 rpm, the journal exhibits period-2 motion regardless of whether turbulent and thermal effects are considered (see Figs. 10.3 and 11.3), but there exist significant differences, such as in the amplitude and form, between the two orbits. At $8,200 \mathrm{rpm}$, a period-3 motion arises for the journal in the system when turbulent and thermal effects are considered (see Fig. 10.4); however, the motion of the journal is approximately 4-periodic when turbulent and thermal effects are neglected, which can be obtained from the four points of the Poincaré map (see Fig. 11.4). At 8,300 rpm, the journal displays period-7 motion when turbulent and thermal effects are included (see Fig. 10.5), while a period-2 motion arises for the journal when turbulent and thermal effects are ignored (see 


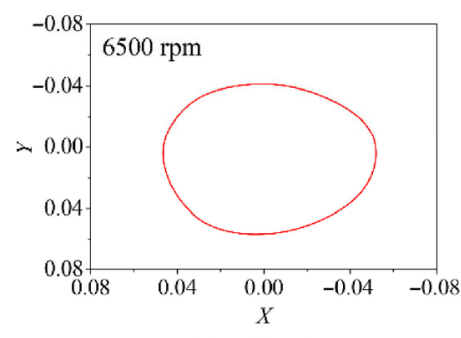

Fig. 10.1 (a)

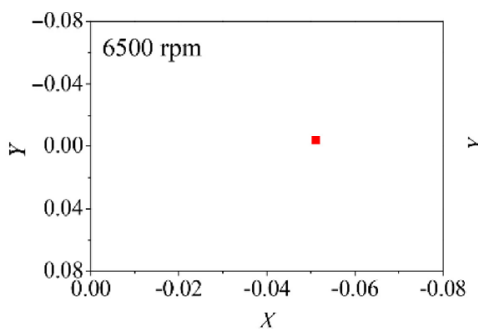

Fig. 10.1 (b)

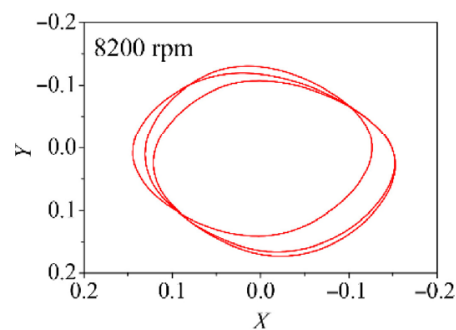

Fig. 10.4 (a)

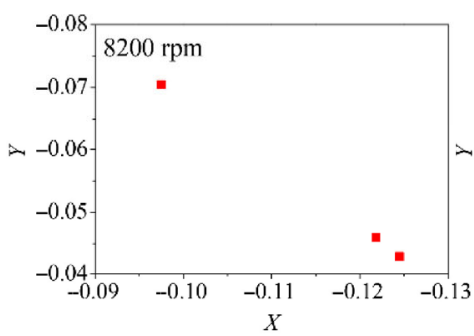

Fig. 10.4 (b)

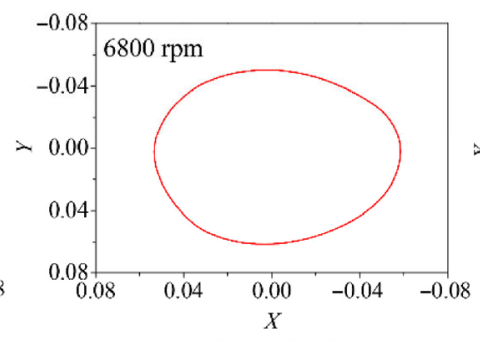

Fig. 10.2 (a)

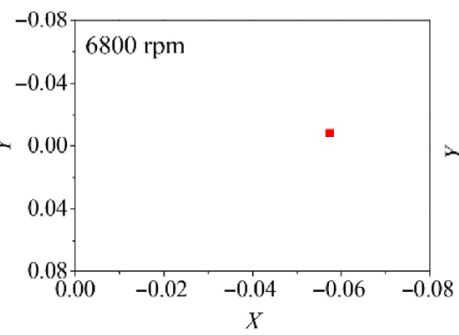

Fig. 10.2 (b)

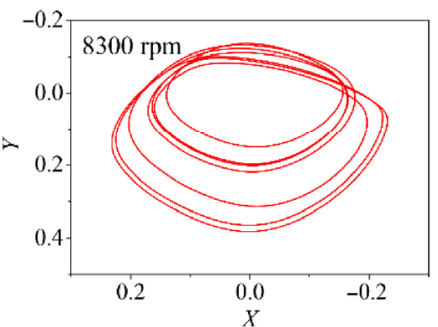

Fig. 10.5 (a)

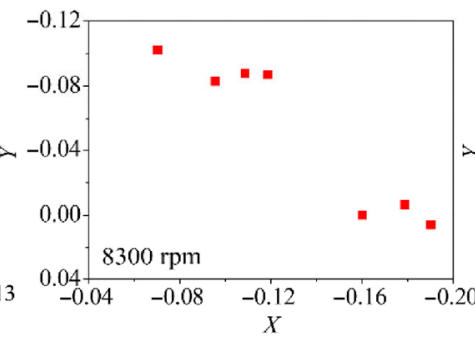

Fig. 10.5 (b)

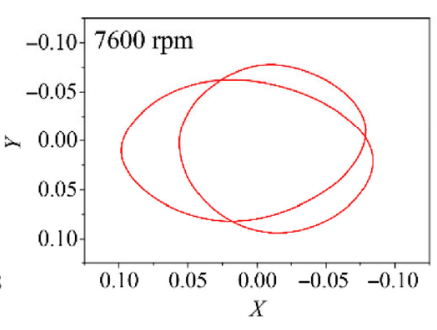

Fig. 10.3 (a)

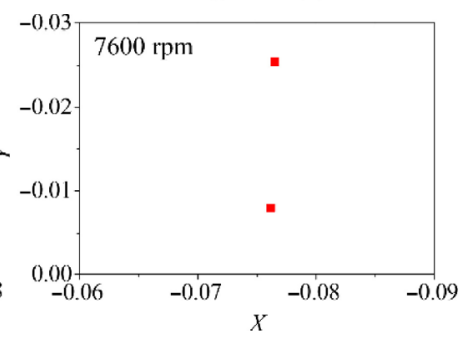

Fig. 10.3 (b)

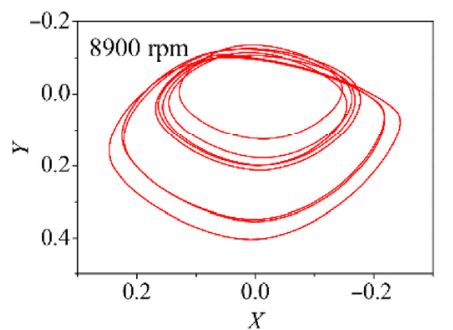

Fig. 10.6 (a)

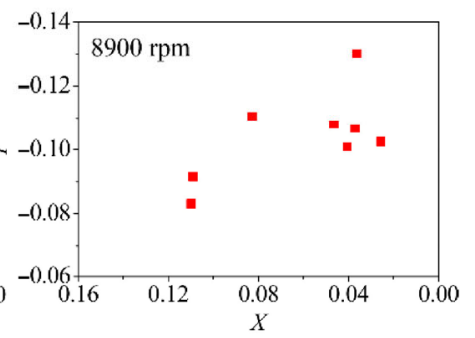

Fig. 10.6 (b)

Fig. 10 (a) Journal center orbits; (b) Poincaré maps at 6,500 rpm, 6,800 rpm, 7,600 rpm, 8,200 rpm, 8,300 rpm, and 8,900 rpm with turbulent and thermal effects.

Fig. 11.5). At 8,900 rpm, a period-8 solution is obtained for the journal when turbulent and thermal effects are considered (see Fig. 10.6). However, in Fig. 11.6, the motion trajectory is highly disordered and the Poincaré mapping points are clustered in groups; thus, the journal exhibits chaotic motion when turbulent and thermal effects are neglected.

\subsection{Vibration response of four pads with and without turbulent and thermal effects}

Figures 12 and 13 show the time domain waveforms and the spectrograms for the four pads at $6,500 \mathrm{rpm}$ with and without turbulent and thermal effects, respectively. The frequency ratio in the spectrogram refers to the ratio of pad vibration frequency to rotor rotational frequency. As shown in Fig. 12, when turbulent and thermal effects are considered, the motions of the four pads are all synchronous, and the vibration frequencies of the four pads primarily match the rotor rotational frequency. Figure 13 shows that when turbulent and thermal effects are neglected, a subsynchronous vibration of the four pads arises in the system, which can be observed from three fluttering frequency components (frequency ratios of 1/4, 1/2, and $3 / 4$ ) lower than the rotor rotational frequency in 


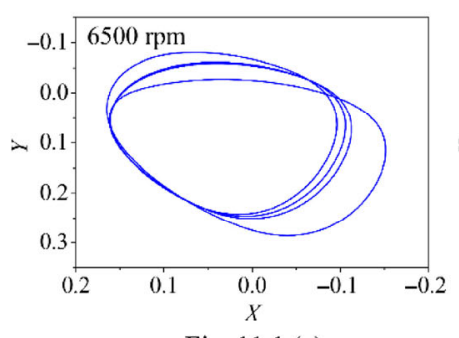

Fig. 11.1 (a)

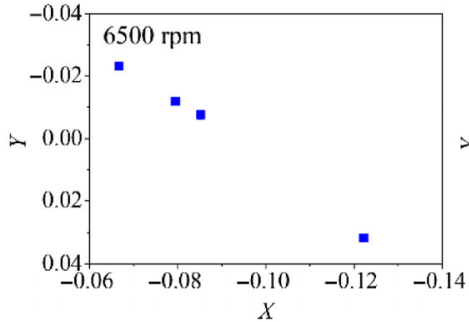

Fig. 11.1 (b)

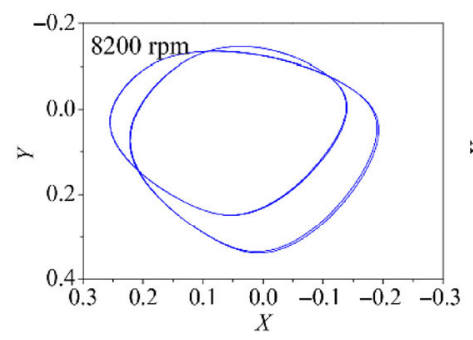

Fig. 11.4 (a)

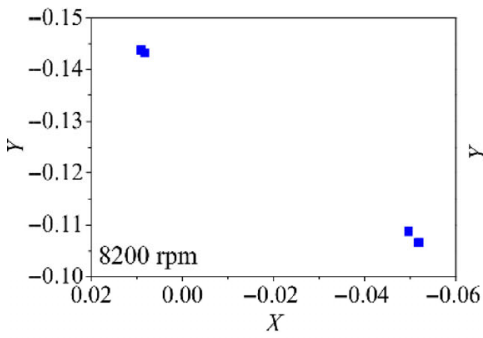

Fig. 11.4 (b)

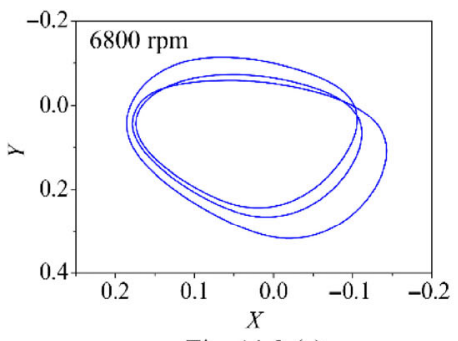

Fig. 11.2 (a)

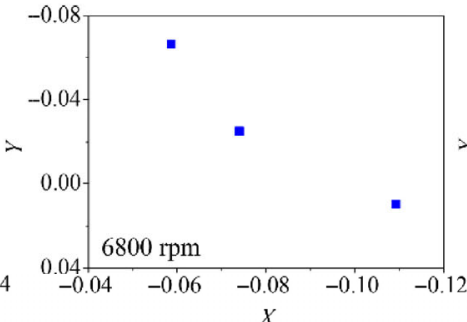

Fig. 11.2 (b)

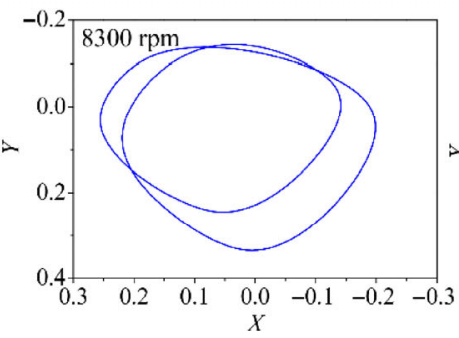

Fig. 11.5 (a)

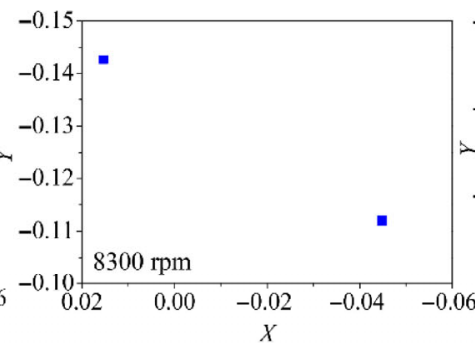

Fig. 11.5 (b)

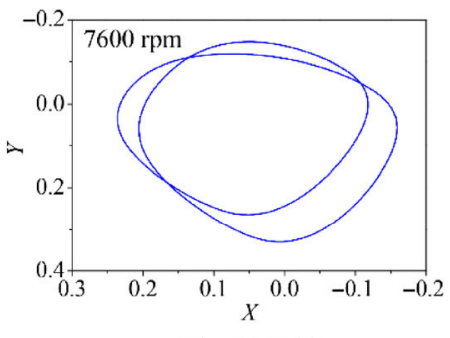

Fig. 11.3 (a)

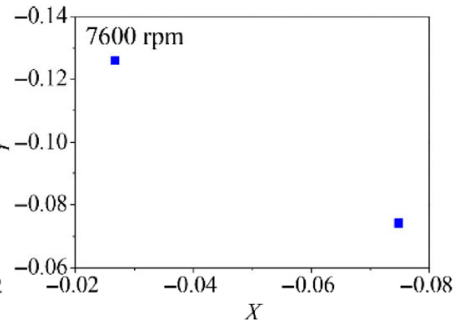

Fig. 11.3 (b)

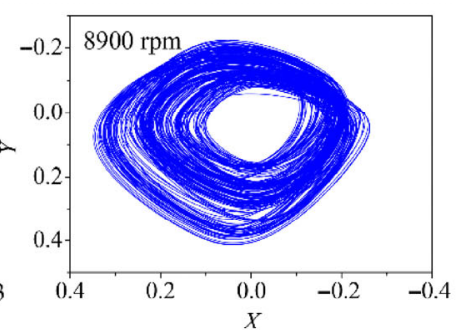

Fig. 11.6 (a)

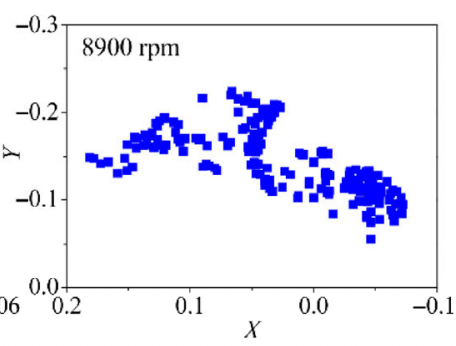

Fig. 11.6 (b)

Fig. 11 (a) Journal center orbits; (b) Poincaré maps at 6,500 rpm, 6,800 rpm, 7,600 rpm, 8,200 rpm, 8,300 rpm, and 8,900 rpm without turbulent or thermal effects.

the spectrograms.

Figures 14 and 15 show time domain waveforms and spectrograms for the four pads at 8,900 rpm with and without turbulent and thermal effects, respectively. Figure 14 shows that when turbulent and thermal effects are considered, seven obvious fluttering frequency components arise (frequency ratios of 1/8, 1/4, $3 / 8,1 / 2,5 / 8,3 / 4$, and $7 / 8$ ) in the spectrograms, indicating that the subsynchronous vibration of the four pads is stronger and more complex than that at 6,500 rpm. As shown in Fig. 15, when turbulent and thermal effects are ignored, there exist numerous irregular, continuous, and broadband frequency components in the spectrograms in addition to the main rotor's rotational frequency; thus, chaotic vibrations may occur for the four pads in the system.

\section{Conclusions}

The current work builds upon the DM to calculate the NFFF of a TPJB while considering turbulent and thermal effects. An NFFF database with high accuracy and large capacity is compiled, and a combined linear and parabolic interpolation polynomial of the NFFF 


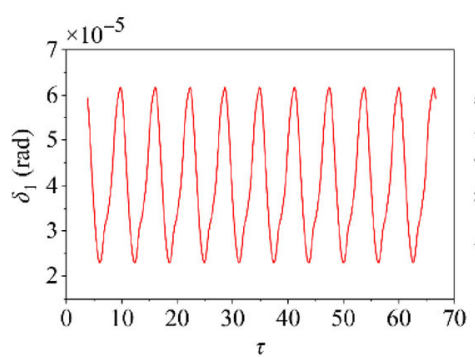

Fig. 12.1 (a)

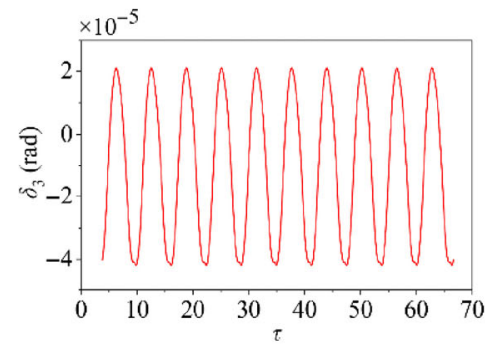

Fig. 12.3 (a)

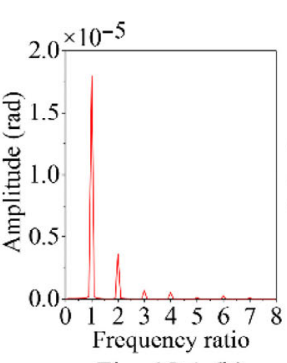

Fig. 12.1 (b)

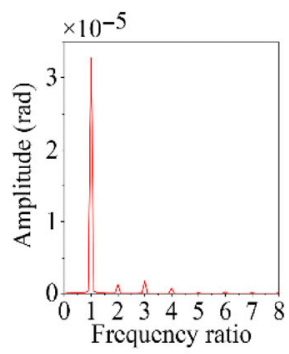

Fig. 12.3 (b)

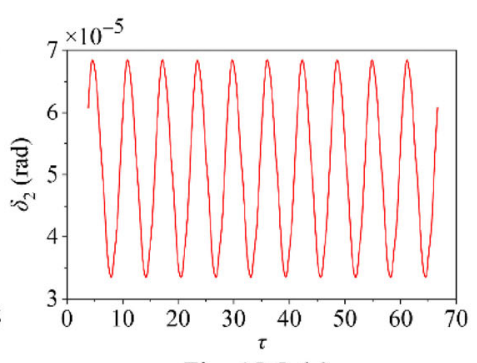

Fig. 12.2 (a)

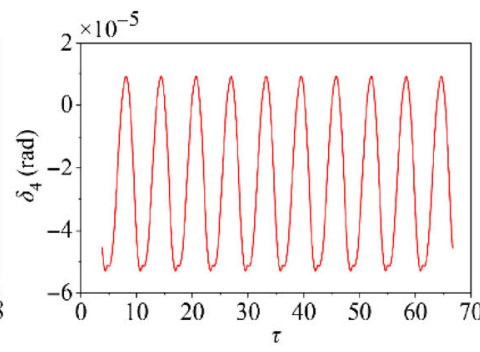

Fig. 12.4 (a)

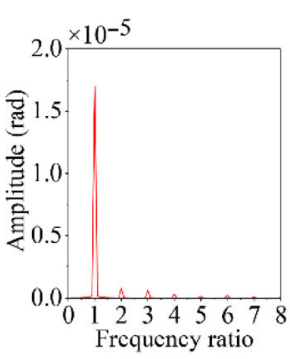

Fig. 12.2 (b)

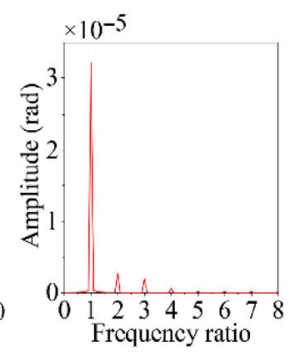

Fig. 12.4 (b)

Fig. 12 (a) Time domain waveforms; (b) spectrograms of the four pads at 6,500 rpm with turbulent and thermal effects.

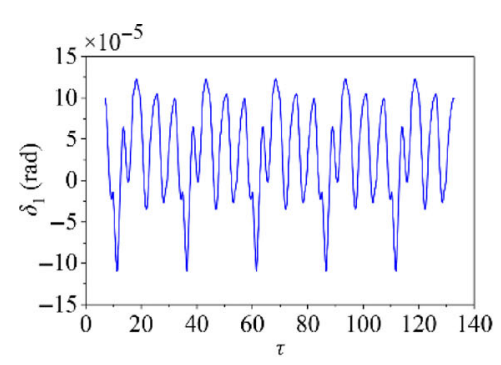

Fig. 13.1 (a)

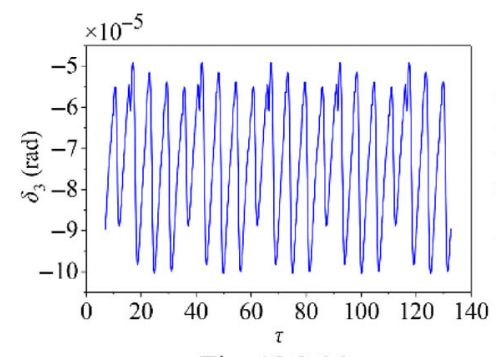

Fig. 13.3 (a)

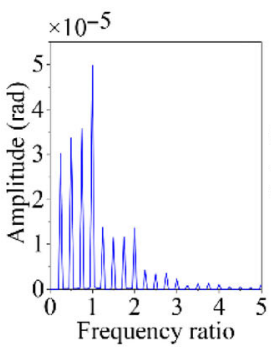

Fig. 13.1 (b)

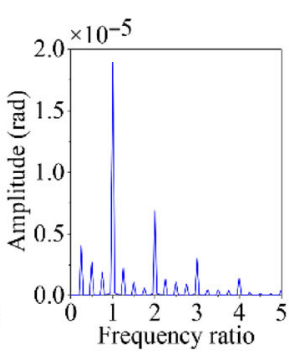

Fig. 13.3 (b)

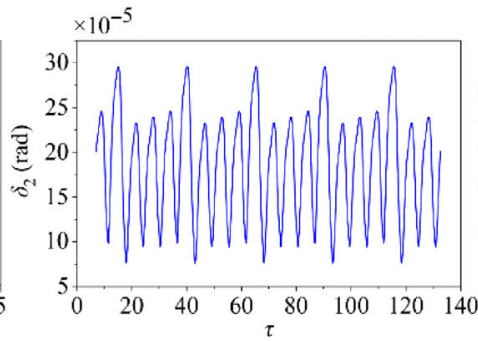

Fig. 13.2 (a)

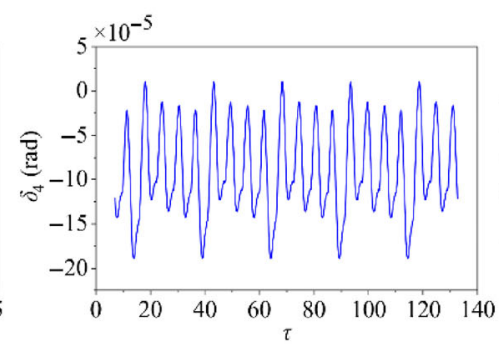

Fig. 13.4 (a)

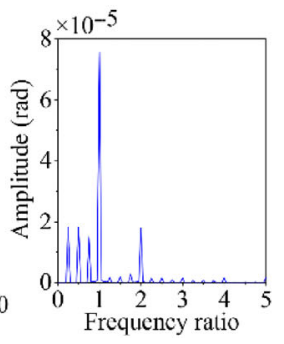

Fig. 13.2 (b)

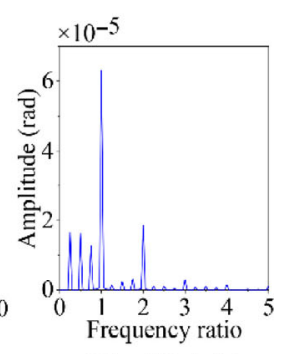

Fig. 13.4 (b)

Fig. 13 (a)Time domain waveforms; (b) spectrograms of the four pads at 6,500 rpm without turbulent or thermal effects.

for a single pad is constructed. The DM is applied to perform nonlinear dynamic calculations of a waterlubricated TPJB-rotor system. The results show that the present DM solution is in good agreement with the FDM solution when turbulent and thermal effects are considered and agrees with the previous DM solution when considering laminar and isothermal effects. Significant differences arise in the results with respect to nonlinear dynamic behavior, such as synchronous motion, period-doubling or sub- synchronous motion, and chaotic motion, for a water-lubricated TPJB-rotor system with and without turbulent and thermal effects. For synchronous motion, the journal center orbit is closer to the TPJB center, and the orbit amplitude is generally smaller when considering turbulent and thermal effects compared with the results obtained without turbulent or thermal effects. These differences are primarily caused by the flow regime, rather than the temperature increase of the water film. 


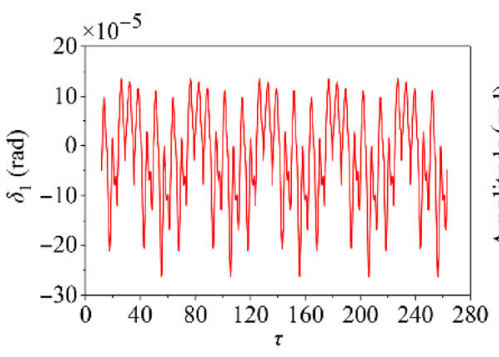

Fig. 14.1 (a)

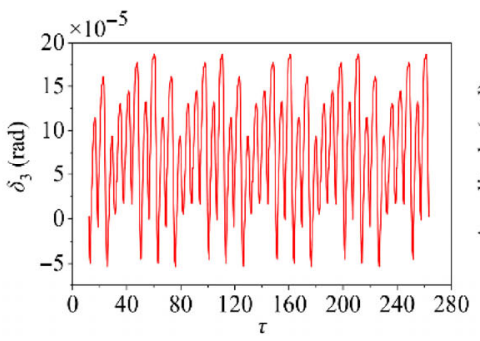

Fig. 14.3 (a)

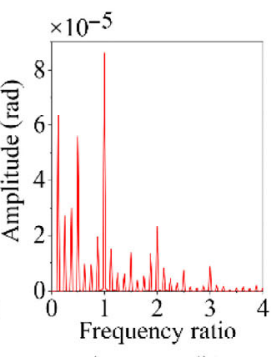

Fig. 14.1 (b)

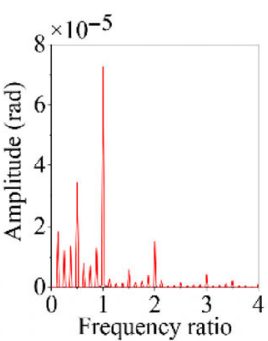

Fig. 14.3 (b)

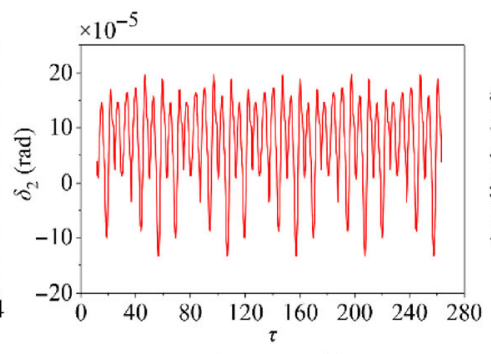

Fig. 14.2 (a)

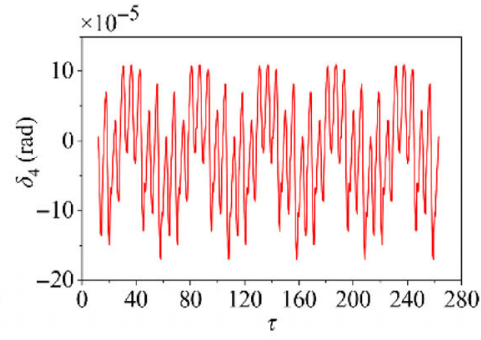

Fig. 14.4 (a)

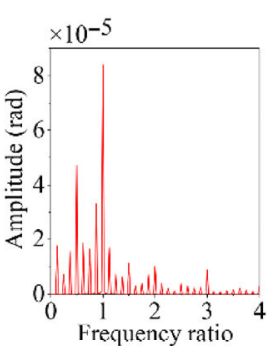

Fig. 14.2 (b)

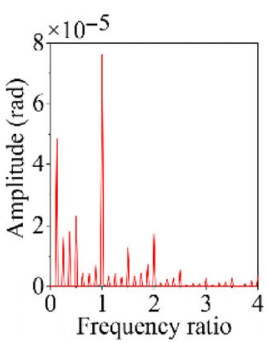

Fig. 14.4 (b)

Fig. 14 (a) Time domain waveforms; (b) spectrograms of the four pads at 8,900 rpm with turbulent and thermal effects.

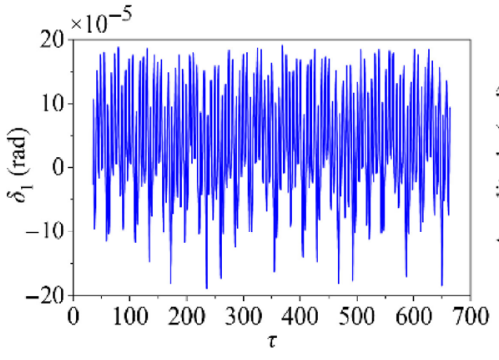

Fig. 15.1 (a)

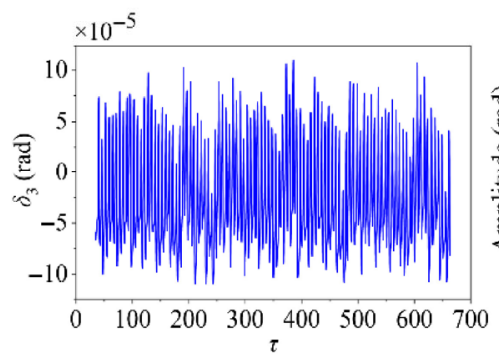

Fig. 15.3 (a)

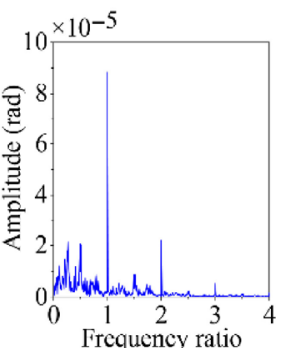

Fig. 15.1 (b)

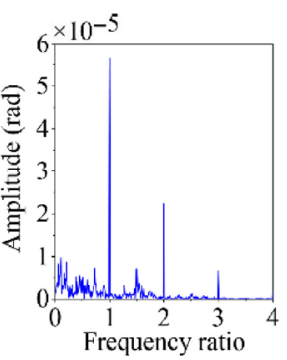

Fig. 15.3 (b)

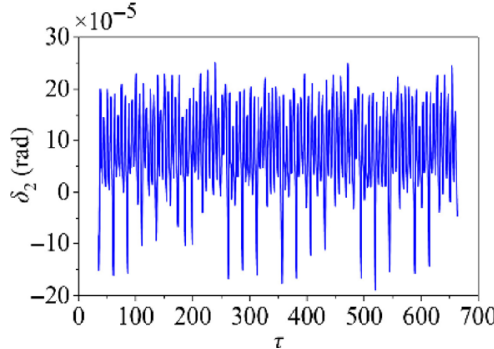

Fig. 15.2 (a)

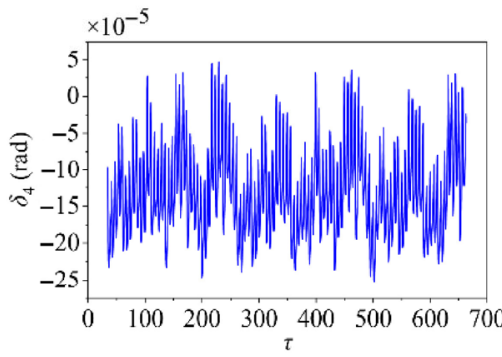

Fig. 15.4 (a)

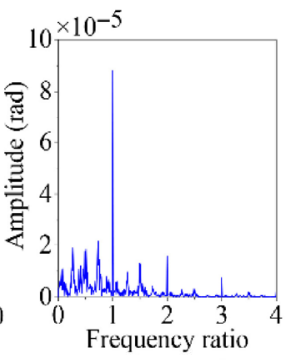

Fig. $15.2(\mathrm{~b})$

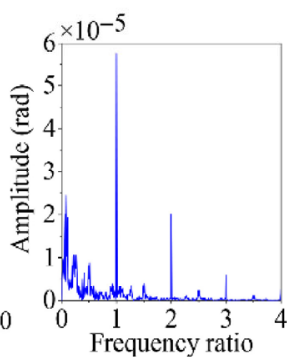

Fig. 15.4 (b)

Fig. 15 (a) Time domain waveforms; (b) spectrograms of the four pads at 8,900 rpm without turbulent or thermal effects.

\section{Acknowledgements}

This work was supported by the National Basic Research Program of China (Grant No. 2015CB057303) and the National Natural Science Foundation of China (Grant No. 51775412).

\section{Appendix}

The ratio of a normal variable to a dimensionless variable is called a dimensional ratio. In this paper, the dimensional ratios are $c$ for $X, Y, H, \varepsilon_{\mathrm{d}}$, and $\varepsilon ; \omega c$ for $\varepsilon^{\prime}$ and $\varepsilon \theta^{\prime} ; \omega^{2} c$ for $X^{\prime \prime}, Y^{\prime \prime}$, and $G ; \mu_{0} L /\left(\omega \psi^{3}\right)$ for $M_{\mathrm{j}}$ and $M_{\mathrm{d}} ; 2 \mu_{0} \omega L / \psi^{3}$ for $K$ and $K_{\delta} ; \mu_{0} \omega R L / \psi^{2}$ for $F X$ and $F Y ; 1 / \omega$ for $\tau ; \mu_{0} \omega R^{2} L / \psi^{2}$ for $\bar{M}_{i} ; \mu_{0} R^{2} L /\left(\omega \psi^{2}\right)$ for $\bar{J}$; $2 \mu_{0} L / \psi^{3}$ for $C_{\delta} ; \omega$ for $\delta_{i}{ }^{\prime} ; \omega^{2}$ for $\delta_{i}{ }^{\prime \prime} ; 2 \mu_{0} \omega / \psi^{2}$ for $P ; \mu_{0}$ for $\bar{\mu} ; L / 2$ for $\lambda$; and $\mu_{0} \omega /\left(\rho c_{v} \psi^{2}\right)$ for $\bar{T}$, where $c_{v}$ is the fluid specific heat.

$\bar{\varepsilon}, \bar{\theta}, \bar{\varepsilon}^{\prime}$, and $\bar{\varepsilon} \bar{\theta}^{\prime}$ can be derived as follows: 


$$
\begin{aligned}
& \bar{\varepsilon}=\sqrt{\left(\begin{array}{l}
\left.\varepsilon \cos \theta+\frac{\delta_{i}}{\psi} \sin \beta-A \cos \beta\right)^{2} \\
+\left(\varepsilon \sin \theta-\frac{\delta_{i}}{\psi} \cos \beta-A \sin \beta\right)^{2}
\end{array}\right.} \\
& \left\{\begin{array}{l}
\cos \bar{\theta}=\left(\varepsilon \cos \theta+\frac{\delta_{i}}{\psi} \sin \beta-A \cos \beta\right) / \bar{\varepsilon} \\
\sin \bar{\theta}=\left(\varepsilon \sin \theta-\frac{\delta_{i}}{\psi} \cos \beta-A \sin \beta\right) / \bar{\varepsilon}
\end{array}\right. \\
& \bar{\varepsilon}^{\prime}=\left(\varepsilon^{\prime} \cos \theta-\varepsilon \theta^{\prime} \sin \theta+\frac{\delta_{i}^{\prime}}{\psi} \sin \beta\right) \cos \bar{\theta} \\
& +\left(\varepsilon^{\prime} \sin \theta+\varepsilon \theta^{\prime} \cos \theta-\frac{\delta_{i}^{\prime}}{\psi} \cos \beta\right) \sin \bar{\theta} \\
& \bar{\varepsilon} \bar{\theta}^{\prime}=\left(\varepsilon^{\prime} \sin \theta+\varepsilon \theta^{\prime} \cos \theta-\frac{\delta_{i}^{\prime}}{\psi} \cos \beta\right) \cos \bar{\theta} \\
& -\left(\varepsilon^{\prime} \cos \theta-\varepsilon \theta^{\prime} \sin \theta+\frac{\delta_{i}^{\prime}}{\psi} \sin \beta\right) \sin \bar{\theta}
\end{aligned}
$$

This paper takes the first pad as an example to derive the formula of $\bar{\varepsilon}_{\max }(\bar{\theta})$. In the four-pad TPJB, the maximum journal eccentricity ratio $\varepsilon_{\max }$ and the two maximum tilting angles of the pad $\left(\delta_{\max 1}, \delta_{\max 2}\right)$ are

$$
\begin{gathered}
\varepsilon_{\max }=\sqrt{2} \cdot(1-A) \\
\delta_{\max 1}=\frac{\left[A \cos \left(\beta-\phi_{2}\right)-\varepsilon_{\max } \cos \left(\phi_{2}-\pi\right)-1\right] \psi}{\sin \left(\beta-\phi_{2}\right)} \\
\delta_{\max 2}=\frac{\left[A \cos \left(\beta-\phi_{1}\right)-\varepsilon_{\max } \cos \left(\phi_{1}-\pi / 2\right)-1\right] \psi}{\sin \left(\beta-\phi_{1}\right)}
\end{gathered}
$$

Then, the dimensionless fluid film thickness at the pivot of the pad is

$$
H_{\mathrm{p}}=1-A+\sqrt{2} \varepsilon_{\max } / 2
$$

The equivalent journal eccentricity ratios $\left(\bar{\varepsilon}_{\max 1}\right.$, $\bar{\varepsilon}_{\max 2}$ ) and the corresponding equivalent journal attitude angles $\left(\bar{\theta}_{1}, \bar{\theta}_{2}\right)$ can be written as

$$
\bar{\varepsilon}_{\max 1}=\sqrt{\left(-\varepsilon_{\max }+\frac{\delta_{\max 1}}{\psi} \sin \beta-A \cos \beta\right)^{2}}
$$

$$
\begin{gathered}
\left\{\begin{array}{l}
\cos \bar{\theta}_{1}=\left(-\varepsilon_{\text {max }}+\frac{\delta_{\max 1}}{\psi} \sin \beta-A \cos \beta\right) / \bar{\varepsilon}_{\max 1} \\
\sin \bar{\theta}_{1}=\left(\frac{\delta_{\max 1}}{\psi} \cos \beta-A \sin \beta\right) / \bar{\varepsilon}_{\max 1}
\end{array}\right. \\
\bar{\varepsilon}_{\max 2}=\sqrt{\left(\begin{array}{l}
\left.\frac{\delta_{\max 2}}{\psi} \sin \beta-A \cos \beta\right)^{2} \\
+\left(\varepsilon_{\max }+\frac{\delta_{\max 2}}{\psi} \cos \beta-A \sin \beta\right)^{2}
\end{array}\right.} \\
\left\{\begin{array}{l}
\cos \bar{\theta}_{2}=\left(\frac{\delta_{\max 2}}{\psi} \sin \beta-A \cos \beta\right) / \bar{\varepsilon}_{\max 2} \\
\sin \bar{\theta}_{2}=\left(\varepsilon_{\max }+\frac{\delta_{\max 2}}{\psi} \cos \beta-A \sin \beta\right) / \bar{\varepsilon}_{\max 2}
\end{array}\right.
\end{gathered}
$$

Thus, $\bar{\varepsilon}_{\max }(\bar{\theta})$ can be derived as follows:

$$
\bar{\varepsilon}_{\max }(\bar{\theta})= \begin{cases}1, & \bar{\theta} \in\left[\phi_{1}+\pi, \phi_{2}+\pi\right) \\ -\frac{1}{\cos \left(\phi_{2}-\bar{\theta}\right)}, & \bar{\theta} \in\left[\phi_{2}+\pi, 2 \pi\right] \cup\left[0, \bar{\theta}_{1}\right) \\ \frac{H_{\mathrm{p}}-1}{\cos (\beta-\bar{\theta})}, & \bar{\theta} \in\left[\bar{\theta}_{1}, \bar{\theta}_{2}\right) \\ -\frac{1}{\cos \left(\phi_{1}-\bar{\theta}\right)}, & \bar{\theta} \in\left[\bar{\theta}_{2}, \phi_{1}+\pi\right)\end{cases}
$$

The moment of inertia of the pad can be derived as

$$
J=\rho_{p} L\left\{\begin{array}{l}
\alpha\left[\frac{1}{2}\left(R+h_{\mathrm{p}}\right)^{2} R^{2}+\frac{1}{4}\left(R+h_{\mathrm{p}}\right)^{4}-\frac{3}{4} R^{4}\right] \\
+\frac{2}{3}\{\sin [\alpha(1-\mathrm{Pc})]+\sin (\alpha \cdot \mathrm{Pc})\} \\
\times\left[R^{4}-\left(R+h_{\mathrm{p}}\right)^{3} R\right]
\end{array}\right\}
$$

where $\rho_{\mathrm{p}}$ and $h_{\mathrm{p}}$ are the pad density and thickness, respectively.

Open Access This article is licensed under a Creative Commons Attribution 4.0 International License, which permits use, sharing, adaptation, distribution and reproduction in any medium or format, as long as you give appropriate credit to the original author(s) and the source, provide a link to the Creative Commons licence, and indicate if changes were made. 
The images or other third party material in this article are included in the article's Creative Commons licence, unless indicated otherwise in a credit line to the material. If material is not included in the article's Creative Commons licence and your intended use is not permitted by statutory regulation or exceeds the permitted use, you will need to obtain permission directly from the copyright holder.

To view a copy of this licence, visit http://creativecommons.org/licenses/by/4.0/.

\section{References}

[1] Wang J K, Khonsari M M. Effects of oil inlet pressure and inlet position of axially grooved infinitely long journal bearings. Part I: Analytical solutions and static performance. Tribol Int 41(2): 119-131 (2008)

[2] Wang J K, Khonsari M M. Effects of oil inlet pressure and inlet position of axially grooved infinitely long journal bearings. Part II: Nonlinear instability analysis. Tribol Int 41(2): 132-140 (2008)

[3] Chang-Jian C W. Nonlinear analysis for gear pair system supported by long journal bearings under nonlinear suspension. Mech Mach Theory 45(4): 569-583 (2010)

[4] Avramov K V, Borysiuk O V. Nonlinear dynamics of one disk asymmetrical rotor supported by two journal bearings. Nonlinear Dyn 67(2): 1201-1219 (2012)

[5] Shi M L, Wang D Z, Zhang J G. Nonlinear dynamic analysis of a vertical rotor-bearing system. J Mech Sci Technol 27(1): 9-19 (2013)

[6] Dakel M, Baguet S, Dufour R. Nonlinear dynamics of a support-excited flexible rotor with hydrodynamic journal bearings. J Sound Vib 333(10): 2774-2799 (2014)

[7] Okabe E P, Cavalca K L. Rotordynamic analysis of systems with a non-linear model of tilting pad bearings including turbulence effects. Nonlinear Dyn 57(4): 481-495 (2009)

[8] Zhang W, Xu X F. Modeling of nonlinear oil-film force acting on a journal with unsteady motion and nonlinear instability analysis under the model. Int J Nonlinear Sci Numer Simul 1(3): 179-186 (2000)

[9] Zhao S X, Xu H, Meng G, Zhu J. Stability and response analysis of symmetrical single-disk flexible rotor-bearing system. Tribol Int 38(8): 749-756 (2005)

[10] Xia Z P, Qiao G, Zheng T H, Zhang W. Nonlinear modeling and dynamic analysis of the rotor-bearing system. Nonlinear Dyn 57(4): 559-577 (2009)

[11] Bastani Y, de Queiroz M. A new analytic approximation for the hydrodynamic forces in finite-length journal bearings.
J Tribol 132(1): 014502 (2010)

[12] Vignolo G G, Barilá D O, Quinzani L M. Approximate analytical solution to Reynolds equation for finite length journal bearings. Tribol Int 44(10): 1089-1099 (2011)

[13] Sfyris D, Chasalevris A. An exact analytical solution of the Reynolds equation for the finite journal bearing lubrication. Tribol Int 55(4): 46-58 (2012)

[14] Zhang Y F, Hei D, Liu C, Guo B J, Lu Y J, Müller N. An approximate solution of oil film forces of turbulent finite length journal bearing. Tribol Int 74(4): 110-120 (2014)

[15] Hei D, Lu Y J, Zhang Y F, Liu F X, Zhou C, Müller N. Nonlinear dynamic behaviors of rod fastening rotorhydrodynamic journal bearing system. Arch Appl Mech 85(7): 855-875 (2015)

[16] Abu-Mahfouz I, Adams M L. Numerical study of some nonlinear dynamics of a rotor supported on a three-pad tilting pad journal bearing (TPJB). J Vib Acoust Trans 127(3): 262-272 (2005)

[17] Wang Y L, Gao Y, Cui Y, Liu Z S. Establishment of approximate analytical model of oil film force for finite length tilting pad journal bearings. Int J Rotat Mach 2015: 531209 (2015)

[18] Chen Z B, Jiao Y H, Xia S B, Huang W H, Zhang Z M. An efficient calculation method of nonlinear fluid film forces in journal bearing. Tribol Trans 45(3): 324-329 (2002)

[19] Qin P, Shen Y, Zhu J, Xu H. Dynamic analysis of hydrodynamic bearing-rotor system based on neural network. Int J Eng Sci 43(5-6): 520-531 (2005)

[20] Jin Y Z, Shi Z Y, Hong H L, Zhang F, Yuan X Y. Axiomatic design method for supercritical rotor dynamics integrating nonlinear deep knowledge. Proced CIRP 53: 237-246 (2016)

[21] Ying J Y, Jiao Y H, Chen Z B. Nonlinear dynamics analysis of tilting pad journal bearing-rotor system. Shock Vib 18(1-2): 45-52 (2011)

[22] Lü Y J, Zhang Y F, Yu Y B. Yu L. Nonlinear dynamics of flexible rotor system supported on fixed-tilting pad combination journal bearing. J Central South Univ Technol Eng 18(3): 610-617 (2011)

[23] Hei D, Lu Y J, Zhang Y F, Lu Z Y, Gupta P, Müller N. Nonlinear dynamic behaviors of a rod fastening rotor supported by fixed-tilting pad journal bearings. Chaos Solitons Fractals 69: $129-150$ (2014)

[24] Taylor C M. Turbulent lubrication theory applied to fluid film bearing design. Proc Inst Mech Eng Conf Proc 184(12): 40-47 (1969)

[25] Constantinescu V N. Basic relationships in turbulent lubrication and their extension to include thermal effects. $J$ Lubr Technol 95(2): 147-154 (1973) 


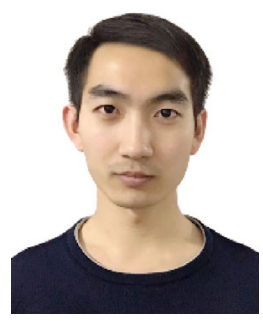

Yingze JIN. He received his bachelor degree in industrial design in 2014 from Zhengzhou University, Zhengzhou, China. After then, he was a Ph.D. student in mechanical

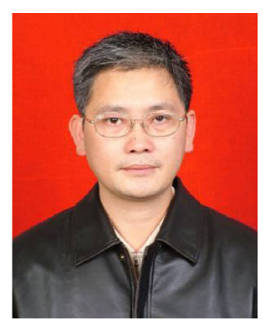

Xiaoyang YUAN. He received his M.S. and Ph.D. degrees in mechanical engineering from $\mathrm{Xi}^{\prime}$ an Jiaotong University, Xi'an, China, in 1985 and 1994, respectively. He is currently a professor with the Key Laboratory engineering at the Key Laboratory of Education Ministry for Modern Design and Rotor-Bearing System, Xi'an Jiaotong University, Xi'an, China. His research interests include hydrodynamic bearings and rotor dynamics.

of Education Ministry for Modern Design and RotorBearing System, Xi'an Jiaotong University. His research areas cover superconducting magnetic technology, modern design and control of electromechanical system, lubrication theory, and dynamics of rotor-bearing system. 